\title{
Taming Tree Amplitudes In General Relativity
}

\author{
Paolo Benincasa \\ Department of Applied Mathematics, University of Western Ontario, London, Ontario \\ N6A 5B\%, Canada \\ Email: pbeninca@uwo.ca
}

\author{
Camille Boucher-Veronneau \\ Department of Physics and Astronomy, University of Waterloo, Waterloo, Ontario N2L \\ 3G1, Canada \\ Perimeter Institute for Theoretical Physics, Waterloo, Ontario N2J 2W9, Canada \\ Email: cboucher-veronneau@perimeterinstitute.ca
}

\section{Freddy Cachazo}

Perimeter Institute for Theoretical Physics, Waterloo, Ontario N2J 2W9, Canada

Email: fcachazo@perimeterinstitute.ca

\begin{abstract}
We give a proof of BCFW recursion relations for all tree-level amplitudes of gravitons in General Relativity. The proof follows the same basic steps as in the BCFW construction and it is an extension of the one given for next-to-MHV amplitudes by one of the authors and P. Svrček in hep-th/0502160. The main obstacle to overcome is to prove that deformed graviton amplitudes vanish as the complex variable parameterizing the deformation is taken to infinity. This step is done by first proving an auxiliary recursion relation where the vanishing at infinity follows directly from a Feynman diagram analysis. The auxiliary recursion relation gives rise to a representation of gravity amplitudes where the vanishing under the BCFW deformation can be directly proven. Since all our steps are based only on Feynman diagrams, our proof completely establishes the validity of BCFW recursion relations. This means that results in the literature that were derived assuming their validity become true statements.
\end{abstract}

KEYwords: Classical Theories of Gravity, Gauge Symmetry. 


\section{Contents}

1. Introduction $\quad 1$

1.1 Preliminaries And Conventions 3

2. BCFW Construction For Gravity Amplitudes 4

3. Vanishing Of $M_{n}(z)$ At Infinity $\quad \mathbf{5}$

3.1 Outline Of The Proof 6

3.2 Auxiliary Recursion Relation $\quad 7$

$\begin{array}{lll}3.3 & \text { Induction And Feynman Diagram Argument } & 10\end{array}$

3.4 Analysis Of The Contribution From Propagators 13

3.4.1 Propagators In Leading Feynman Diagrams Of $M_{\mathcal{I}} \quad 13$

3.4.2 Propagators In Leading Feynman Diagrams Of $M_{\mathcal{J}} \quad 15$

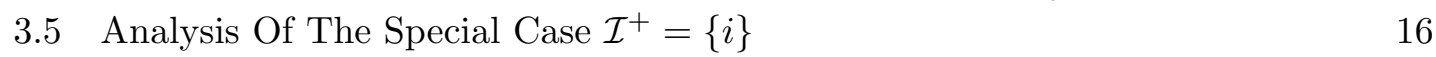

$\begin{array}{ll}\text { 4. Ward Identities } & 18\end{array}$

5. Conclusions And Further Directions 19

A. Proof Of Auxiliary Recursion Relations $\quad 20$

A.1 Vanishing Of $M_{n}(w)$ At Infinity 21

A.2 Location Of Poles And Final Form Of The Auxiliary Recursion Relations 22

B. Large- $z$ behavior of NMHV gravity amplitudes under a three-particle deformation $\quad 22$

\section{Introduction}

The analytic computation of scattering amplitudes in gauge theory and gravity has always been a very challenging problem. In principle, this problem is solved by using Feynman diagrams. However, in practice, the fast growth in the number of diagrams makes the calculation impossible.

In some cases, closed formulas have been found for large classes of amplitudes. One of the main tools has been the use of recursion techniques. Many analytic formulas were found or proven by using the Berends-Giele recursion relations introduced in the 80's [1, $2,3,4,5]$. One important example are the wonderfully simple formulas conjectured by Parke-Taylor [6] for MHV (Maximally Helicity Violating) tree level amplitudes of gluons.

More recently, a new set of recursion relations for tree level amplitudes of gluons was introduced by Britto, Feng and the third author [7]. These recursion relations were inspired 
by $[8,9]$ and reproduced very compact results obtained in [10] by studying the IR behavior of $\mathcal{N}=4$ one-loop amplitudes. A simple and elegant proof of the relations was later given by the same authors in collaboration with Witten in [11]. The proof is constructive and gives rise to a method using the power of complex analysis for deriving similar relations in any theory where physical singularities are well understood. The BCFW method has been successfully applied in many contexts involving massless particles at tree and loop level $[12,13,14,15,16,17,18,19]$ as well as for massive particles at tree level [20].

The possibility of the existence of BCFW recursion relations in General Relativity was first investigated in $[21,22]$. There it was pointed out that the main obstacle to establish the validity of the recursion relations is to prove that deformed amplitudes vanish at infinity while individual Feynman diagrams diverge. In [21], the desired behavior was checked for MHV amplitudes up to $n<11$ under the $(-,-)$ deformation using the BGK formula [23]. In [22], it was shown that the BGK formula vanishes at infinity for any $n^{1}$ under the $(+,-)$ deformation. Also in [22], a proof based on Feynman diagrams was given for all next-toMHV amplitudes ${ }^{2}$ and for all amplitudes up to eight gravitons using the KLT relations [24].

The fact that individual Feynman diagrams diverge very badly in the limit when the deformation parameter is taken to infinity and yet the amplitude vanishes implies that a large number of cancelations must happen. What was shown in [22] is that such cancelations can be made explicit if representations of amplitudes where Feynman diagrams have been re-summed are used.

This is just one more example where Feynman diagrams not only give rise to extremely long answers which then collapse to very compact expressions but actually imply a completely wrong behavior of the amplitude for large momenta.

A surprising example of this, now at the loop level, is the work of $[25,26,27]$ where $\mathcal{N}=8$ supergravity has been shown to possess a remarkably good ultraviolet behavior even though a direct power counting argument indicates that bad divergencies must be present. Also recently, a careful study of the structure of certain one-loop amplitudes in $\mathcal{N}=8$ supergravity shows that even though power counting implies that after a PassarinoVeltman reduction [28] the amplitude should contain boxes, triangles, bubbles and rational pieces only the boxes can have non-zero coefficients [29, 30]. That this might hold for generic one-loop amplitudes is now known as the no-triangle hypothesis [29, 30]. A striking possibility, which could explain all these properties, is that a twistor string-like construction for this theory could exists [31, 32, 33].

In this paper we give a complete proof that the miraculous behavior exhibited in next-to-MHV tree level amplitudes of gravitons in [22] actually extends to all amplitudes.

The strategy we follow is exactly the same as the one used in [22] to prove the nextto-MHV case. We use an auxiliary recursion relation to derive a more convenient representation for the amplitudes and then show that they vanish at infinity under the BCFW deformation.

\footnotetext{
${ }^{1}$ Since the BGK formula has been tested against Feynman diagrams only for $n<11$ [23], one cannot make a general statement for actual amplitudes based on BGK.

${ }^{2}$ Although not mentioned in [22], this technique clearly also works for MHV amplitudes.
} 
The most important aspect of our proof is that both the auxiliary recursion relations and the vanishing under the BCFW deformation are proven using only Feynman diagram arguments. Since Feynman diagrams are the basic way to define gravity amplitudes, our result completely establishes the validity of the BCFW recursion relations for General Relativity.

The auxiliary recursion relations are obtained by using a deformation that affects the maximum possible number of polarization tensors while keeping propagators linear functions in the deformation parameter. Such a deformation was also introduced in [22]. Quite interestingly, this "maximal" deformation on a given amplitude induces non-maximal deformations on amplitudes with smaller number of gravitons. One of the non-maximal deformations that naturally shows up only affects gravitons of a given helicity. Very interesting results have been obtained in the literature by assuming that under such deformations amplitudes vanish at infinity. More precisely, Bjerrum-Bohr et.al were able to derive MHV expansions for gravity in [34] along the same lines as done for gauge theory by Risager in [35]. As mentioned in [34], a proof of the validity of the basic BCFW deformation will constitute evidence for the validity of the non-maximal deformations as some of them can be thought of as compositions of the basic one ${ }^{3}$.

It is also important to mention that at one-loop in gauge theory one can find that compositions of BCFW deformations can vanish at infinity while individual deformations do not. This was actually the motivation for the first use of compositions in the literature in $[37]$.

This paper is organized as follows: In section II, we follow the same steps as in the original BCFW construction to show the form of recursion relations for gravity amplitudes that can be obtained if one assumes that the amplitudes vanish at infinity under the deformation. In section III we prove that statement by using auxiliary recursion relations. In section IV, we use Ward identities for MHV amplitudes to show how our proof implies the validity of other recursion relations obtained by different deformations. In section $\mathrm{V}$ we give our conclusions and future directions. Part of the proof of the validity of the auxiliary recursion relations is given in the appendix.

\subsection{Preliminaries And Conventions}

Tree level amplitudes of gravitons are rational functions of the momenta of the gravitons and multilinear functions of the polarization tensors. It is convenient to encode all the information in terms of spinor variables using the spinor-helicity formalism [38, 39, 40]. Each momentum vector can be written as a bispinor $p_{a \dot{a}}=\lambda_{a} \tilde{\lambda}_{\dot{a}}$. We define the inner product of spinors as follows $\left\langle\lambda, \lambda^{\prime}\right\rangle=\epsilon^{a b} \lambda_{a} \lambda_{b}^{\prime}$ and $\left[\tilde{\lambda}, \tilde{\lambda}^{\prime}\right]=\epsilon^{\dot{a} \dot{b}} \tilde{\lambda}_{\dot{a}} \tilde{\lambda}_{\dot{b}}^{\prime}$. Polarization tensors of gravitons can be expressed in terms of polarization vectors of gauge bosons as follow

$$
\epsilon_{a \dot{a}, b \dot{b}}^{+}=\epsilon_{a \dot{a}}^{+} \epsilon_{b \dot{b}}^{+}, \quad \epsilon_{a \dot{a}, b \dot{b}}^{-}=\epsilon_{a \dot{a}}^{-} \epsilon_{b \dot{b}}^{-}
$$

\footnotetext{
${ }^{3}$ After the first version of this paper was submitted, [36] found by direct numerical analysis that nonmaximal deformations in gravity generically fail for $n \geq 12$. This means that the MHV expansion for gravity cannot be obtained in the way proposed in [34]. See the note added for more details and appendix B for a proof.
} 
where polarization vectors of gauge bosons are given by

$$
\epsilon_{a \dot{a}}^{+}=\frac{\mu_{a} \tilde{\lambda}_{\dot{a}}}{\langle\mu, \lambda\rangle}, \quad \epsilon_{a \dot{a}}^{-}=\frac{\lambda_{a} \tilde{\mu}_{\dot{a}}}{[\tilde{\lambda}, \tilde{\mu}]}
$$

with $\mu_{a}$ and $\tilde{\mu}_{\dot{a}}$ arbitrary reference spinors.

Using the spinor-helicity formalism all the information about a particular graviton is encoded in $\lambda, \tilde{\lambda}$ and the helicity, $h$, which can be positive or negative. Therefore a given amplitude can be written as

$$
\mathcal{M}_{n}\left(1^{h_{1}}, \ldots, n^{h_{n}}\right)=\kappa^{n-2} \delta^{(4)}\left(\sum_{i=1}^{n} \lambda_{a}^{(i)} \tilde{\lambda}_{\dot{a}}^{(i)}\right) M_{n}\left(1^{h_{1}}, \ldots, n^{h_{n}}\right),
$$

where $\kappa^{2}=8 \pi G_{\mathrm{N}}$, the label $(i)$ on the spinors is the particle label and the notation $\left(i^{h_{i}}\right)$ stands for $\left(\lambda^{(i)}, \tilde{\lambda}^{(i)}, h_{i}\right)$. In the rest of this paper we will only be concerned with $M_{n}\left(1^{h_{1}}, \ldots, n^{h_{n}}\right)$.

Sometimes it will be convenient to write $\left(i^{h_{i}}\right)$ as $p_{i}^{h_{i}}$ where $p_{i}$ is the momentum of the $i^{\text {th }}$ graviton.

Also useful is the following notation: $\left\langle\lambda|P| \tilde{\lambda}^{\prime}\right]=-\lambda^{a} P_{a \dot{a}} \tilde{\lambda}^{\dot{a}}$. The minus sign in the definition is there so that if $P_{a \dot{a}}$ is a null vector $\mu_{a} \tilde{\mu}_{\dot{a}}$ one has $\left\langle\lambda|P| \tilde{\lambda}^{\prime}\right]=\langle\lambda, \mu\rangle[\tilde{\mu}, \tilde{\lambda}]$. This formula has several generalizations. In this paper we only use the one that involves two generic vectors $P$ and $Q$ that are written as sums of null vectors as $P=\sum_{s} p_{s}$ and $Q=\sum_{r} p_{r}$. Then we have

$$
\left\langle\lambda|P Q| \lambda^{\prime}\right\rangle=\sum_{r, s}\left\langle\lambda, \lambda^{(r)}\right\rangle\left[\tilde{\lambda}^{(r)}, \tilde{\lambda}^{(s)}\right]\left\langle\lambda^{(s)}, \lambda^{\prime}\right\rangle .
$$

\section{BCFW Construction For Gravity Amplitudes}

Consider a scattering amplitude of $n$ gravitons $M_{n}\left(1^{h_{1}}, \ldots, n^{h_{n}}\right)$. Construct a one complex parameter deformation of the amplitude that preserves the physical properties of being on-shell and momentum conservation. The simplest way to achieve this is by choosing two gravitons of opposite helicities ${ }^{4}$, say $i^{+}$and $j^{-}$, and perform the following deformation

$$
\lambda^{(i)}(z)=\lambda^{(i)}+z \lambda^{(j)}, \quad \tilde{\lambda}^{(j)}(z)=\tilde{\lambda}^{(j)}-z \tilde{\lambda}^{(i)} .
$$

All other spinors remain the same. The deformation parameter $z$ is a complex variable. It is easy to check that this deformation preserves the on-shell conditions of all gravitons, i.e., $p_{k}(z)^{2}=0$ for any $k$ and momentum conservation since $p_{i}(z)+p_{j}(z)=p_{i}+p_{j}$.

The main observation is that the scattering amplitude is a rational function of $z$ which we denote by $M_{n}(z)$. This fact follows from $M_{n}\left(1^{h_{1}}, \ldots, n^{h_{n}}\right)$ being a rational function of momenta and polarization tensors. Being a rational function of $z, M_{n}(z)$ can be determined if complete knowledge of its poles, residues and behavior at infinity is found.

\footnotetext{
${ }^{4}$ This is always possible since tree-level amplitudes with all equal helicities vanish and are not of interest for our discussion.
} 
We claim that $M_{n}(z)$ only has simple poles and it vanishes as $z$ is taken to infinity. This means that

$$
M_{n}(z)=\sum_{\alpha} \frac{c_{\alpha}}{z-z_{\alpha}}
$$

where the sum is over all poles of $M_{n}(z)$.

The fact that $M_{n}(z)$ only has simple poles follows by considering its form as a sum over Feynman diagrams. Choosing a gauge where polarization tensors do not have poles in $z$, i.e, one in which the reference spinors of the $i^{\text {th }}$ and $j^{\text {th }}$ gravitons are $\mu_{a}=\lambda_{a}^{(j)}$ and $\tilde{\mu}_{\dot{a}}=\tilde{\lambda}_{\dot{a}}^{(i)}$ respectively, the only possible singularities come from propagators. Propagators are functions of momenta of the form

$$
\frac{1}{P_{\mathcal{I}}^{2}}=\frac{1}{\left(\sum_{k \in \mathcal{I}} p_{k}\right)^{2}}
$$

where $\mathcal{I} \subset\{1,2, \ldots, n\}$ is some subset of gravitons with more than one and less than $n-1$ elements.

Clearly, the only propagators that can depend on $z$ are those for which either $i \in \mathcal{I}$ or $j \in \mathcal{I}$ but not both. Without loss of generality let us assume that $i \in \mathcal{I}$. Then the propagator has the form

$$
\frac{1}{P_{\mathcal{I}}^{2}(z)}=\frac{1}{P_{\mathcal{I}}^{2}(0)-z\left\langle j\left|P_{\mathcal{I}}(0)\right| i\right]}
$$

This shows that all singularities are simple poles. Their location is given by

$$
z_{\mathcal{I}}=\frac{P_{\mathcal{I}}^{2}(0)}{\left\langle j\left|P_{\mathcal{I}}(0)\right| i\right]} .
$$

The proof that $M_{n}(z)$ vanishes as $z$ is taken to infinity is basically the main result of this paper and it is presented in the next section. Here we simply assume it and continue in order to present the final form the BCFW recursion relations.

The final step is the computation of the residues $c_{\mathcal{I}}$. This is easily done since close to the region where a given propagator goes on-shell the amplitude factorizes as the product of lower amplitudes. Collecting all these results one finds that

$$
M_{n}(z)=\sum_{\mathcal{I}, \mathcal{J}} \sum_{h= \pm} M_{\mathcal{I}}\left(\left\{K_{\mathcal{I}}\right\}, p_{i}\left(z_{\mathcal{I}}\right),-P_{\mathcal{I}}^{h}\left(z_{\mathcal{I}}\right)\right) \frac{1}{P_{\mathcal{I}}(z)^{2}} M_{\mathcal{J}}\left(\left\{K_{\mathcal{J}}\right\}, p_{j}\left(z_{\mathcal{I}}\right), P_{\mathcal{I}}^{-h}\left(z_{\mathcal{I}}\right)\right)
$$

where $\{\mathcal{I}, \mathcal{J}\}$ is a partition of the set of all gravitons such that $i \in \mathcal{I}$ and $j \in \mathcal{J}, K_{\mathcal{I}}\left(K_{\mathcal{J}}\right)$ is the collection of all gravitons in $\mathcal{I}(\mathcal{J})$ except for $i(j)$ and $h$ is the helicity of the internal graviton.

The BCFW recursion relation is obtained by setting $z=0$ in (2.6). It is important to mention that the value of $z_{\mathcal{I}}$ was determined by requiring $P_{\mathcal{I}}\left(z_{\mathcal{I}}\right)$ be a null vector. Therefore the BCFW recursion relations only involve physical on-shell amplitudes.

\section{Vanishing Of $M_{n}(z)$ At Infinity}

In the previous section we showed that the validity of the BCFW recursion relations for gravity amplitudes simply follows from the vanishing of $M_{n}(z)$ at infinity. In this section we provide a proof of this statement. 
It is instructive to start by computing what the behavior of $M_{n}(z)$ for large $z$ is from a naive Feynman diagram analysis ${ }^{5}$. A generic Feynman diagram is schematically given by the product of polarization tensors, propagators and vertices. We are looking for Feynman diagrams that give the leading behavior for large $z$. We choose generic reference spinors in polarization tensors such that

$$
\epsilon_{i}^{++}(z)_{a \dot{a}, b \dot{b}} \sim \frac{1}{z^{2}} \frac{\mu_{a} \tilde{\lambda}_{\dot{a}}^{(i)} \mu_{b} \tilde{\lambda}_{\dot{b}}^{(i)}}{\left\langle\mu, \lambda^{(j)}\right\rangle^{2}}, \quad \epsilon_{j}^{--}(z)_{a \dot{a}, b \dot{b}} \sim \frac{1}{z^{2}} \frac{\lambda_{a}^{(j)} \tilde{\mu}_{\dot{a}} \lambda_{b}^{(j)} \tilde{\mu}_{\dot{b}}}{\left[\tilde{\lambda}^{(i)}, \tilde{\mu}\right]^{2}},
$$

while all others are independent of $z$. Note that only vertices that depend on momenta can give $z$ contributions in the numerator. Therefore we should look for Feynman diagrams with the maximum number of $z$ dependent vertices. Such diagrams are those for which one has only cubic vertices. For $n$ gravitons there can be a maximum of $n-2$ vertices. Each vertex can give at most a $z^{2}$ dependence $^{6}$. Therefore, the leading diagrams will have a $z^{2(n-2)}$ dependence from vertices. Finally we are left with propagators. The $z$ dependence flows in the diagram along a unique path connecting the $i^{\text {th }}$ graviton with the $j^{\text {th }}$ graviton. Therefore there are $n-3$ of them. Each propagator gives a $1 / z$ contribution. Collecting all contributions gives

$$
M_{n}(z) \sim\left(\frac{1}{z^{4}}\right)\left(z^{2(n-2)}\right)\left(\frac{1}{z^{(n-3)}}\right)=z^{n-5} .
$$

This implies that $M_{n}(z) \sim 0$ for large $z$ only if $n<5$. As $n$ increases individual Feynman diagrams diverge more at infinity.

This means that we have to find a better representation of $M_{n}(z)$ where Feynman diagrams have been re-summed into better behaved objects. This is the main strategy of our proof.

The proof is straightforward but it might be somewhat confusing if an overall picture is not kept in mind. This is why we first provide an outline and then give the details.

\subsection{Outline Of The Proof}

We start by finding a convenient representation of $M_{n}(z)$. The new representation comes from some auxiliary recursion relations. The auxiliary recursion relations are obtained using a BCFW-like construction but with a deformation under which individual Feynman diagrams vanish at infinity. The way we achieve this is by making as many polarization tensors go to zero at infinity as possible.

Let us denote the new deformation parameter $w$. Then one has that $M_{n}(w) \rightarrow 0$ as $w \rightarrow \infty$. The recursion relations are schematically of the form

$$
M_{n}=\sum_{\mathcal{I}, \mathcal{J}} \sum_{h= \pm} M_{\mathcal{I}}^{h}\left(w_{\mathcal{I}}\right) \frac{1}{P_{\mathcal{I}}^{2}} M_{\mathcal{J}}^{-h}\left(w_{\mathcal{I}}\right)
$$

\footnotetext{
${ }^{5}$ The reason we use the word "naive" is that the argument only takes into account the behavior of individual diagrams and does not consider possible cancelations among them.

${ }^{6}$ This and all statements about the general structure of Feynman diagrams can be easily derived from the lagrangian density $\mathcal{L}=\sqrt{-g} R$ with $g_{\mu \nu}=\eta_{\mu \nu}+h_{\mu \nu}$.
} 
where the sum is over some sets $\mathcal{I}, \mathcal{J}$ of gravitons. These auxiliary recursion relations actually provide the first example of recursion relations valid for all physical amplitudes of gravitons. However, the price one pays for being able to prove that $M_{n}(w) \rightarrow 0$ as $w \rightarrow \infty$ directly from Feynman diagrams is that the number of terms in (3.3) is very large and many of the gravitons depend on $w_{\mathcal{I}}$. These features make (3.3) not very useful for actual computations.

The next step in our proof is to apply the BCFW deformation to $M_{n}$ now given by (3.3). Then we have

$$
M_{n}(z)=\sum_{\{i, j\} \subset \mathcal{J}} \sum_{h= \pm} M_{\mathcal{I}}^{h}\left(w_{\mathcal{I}}\right) \frac{1}{P_{\mathcal{I}}^{2}} M_{\mathcal{J}}^{-h}\left(w_{\mathcal{I}}, z\right)+\sum_{i \in \mathcal{I}, j \in \mathcal{J}} \sum_{h= \pm} M_{\mathcal{I}}^{h}\left(w_{\mathcal{I}}(z), z\right) \frac{1}{P_{\mathcal{I}}^{2}(z)} M_{\mathcal{J}}^{-h}\left(w_{\mathcal{I}}(z), z\right)
$$

where the $z$ dependence on the right hand side can appear implicitly through $w_{\mathcal{I}}(z)$ as well as explicitly. The first set of terms on the right hand side of (3.4) has both deformed gravitons in $\mathcal{J}$. Therefore, all the $z$ dependence is confined to $M_{\mathcal{J}}$. We then show that $M_{\mathcal{J}}$ is a physical amplitude with less than $n$ gravitons under a BCFW deformation. Therefore, we can use an induction argument to prove that it vanishes as $z \rightarrow \infty$.

For the second set of terms the $z$ dependence appears not only explicitly but also implicitly via $w_{\mathcal{I}}$ in many gravitons. Quite nicely, it turns out that one can show that each one of those terms vanishes as $z$ goes to infinity by using a Feynman diagram analysis similar to the one done at the beginning of this section. The reason for this is again the large number of polarization tensors that pick up a $z$ dependence.

There is a special case that has to be considered separately. This is when there is only one positive helicity graviton in $\mathcal{I}$, i.e., the $i^{\text {th }}$ graviton. We prove the desired behavior at infinity in this case at the end of this section.

\subsection{Auxiliary Recursion Relation}

The auxiliary recursion relations we need are obtained by using a composition of BCFW deformations introduced in [22] and which was used to prove the vanishing of $M_{n}(z)$ for next-to-MHV amplitudes. The basic idea comes form the analysis of Feynman diagrams we performed above. It is clear that the reason individual Feynman diagrams diverge as $z \rightarrow \infty$ for $n \geq 5$ is that the number of propagators and vertices grow in the same way but vertices give an extra power of $z$ which can be compensated by two polarization tensors that depend on $z$ only if $n$ is not too large. The key is then to perform a deformation that will make more polarization tensors contribute.

Recall from the outline of the proof that the deformation parameter is denoted by $w$. The simplest choice is to deform the $\lambda$ 's of all positive helicity gravitons and the $\tilde{\lambda}$ 's of all negative helicity gravitons. This choice will give $1 / w^{2 n}$ from the polarization tensors. This makes $M_{n}(w)$ go at most as $1 / w^{4}$ even without taking into account the propagators. Propagators are now quadratic functions of $w$ and therefore they contribute $1 / w^{2}$ each. This last feature is what makes this choice very inconvenient since every multi-particle singularity of the amplitude will result in two simple poles rather than one. 
We are then looking for a deformation that gives a $w$ dependence to the largest number of gravitons and at the same time keeps all propagators at most linear functions of $w$. The most general such deformation depends on the number of plus and minus helicity gravitons in the amplitude. Let $\left\{r^{-}\right\}$and $\left\{k^{+}\right\}$denote the sets of negative and positive helicity gravitons in the amplitude respectively. Also let $m$ and $p$ be the number of elements in each. Then if $p \geq m$ the deformation is

$$
\tilde{\lambda}^{(j)}(w)=\tilde{\lambda}^{(j)}-w \sum_{s \in\left\{k^{+}\right\}} \alpha^{(s)} \tilde{\lambda}^{(s)}, \quad \lambda^{(k)}(w)=\lambda^{(k)}+w \alpha^{(k)} \lambda^{(j)}, \quad \forall k \in\left\{k^{+}\right\}
$$

where $j$ is a negative helicity graviton and $\alpha^{(k)}$ 's can be arbitrary rational functions of kinematical invariants.

If $m \geq p$ the deformation is

$$
\lambda^{(i)}(w)=\lambda^{(i)}+w \sum_{s \in\left\{r^{-}\right\}} \alpha^{(s)} \lambda^{(s)}, \quad \tilde{\lambda}^{(k)}(w)=\tilde{\lambda}^{(k)}-w \alpha^{(k)} \tilde{\lambda}^{(i)}, \quad \forall k \in\left\{r^{-}\right\}
$$

where $i$ is a positive helicity graviton.

The deformation introduced in [22] to prove the case of next-to-MHV amplitudes corresponds to taking all $\alpha^{(s)}=1$ in (3.5). It turns out that not all choices of $\alpha^{(s)}$ lead to the desired behavior of individual Feynman diagrams at infinity. For example, any choice that removes the $w$ dependence on any single spinor or even on any linear combination of subsets of them will fail. This is usually due to some subtle Feynman diagrams. It is interesting that one has to use precisely the maximal choice. In other words, we have to choose all $\alpha^{(s)}=1$. Given that this is the choice we use in the rest of the paper, we rewrite (3.5) and (3.6) with $\alpha^{(k)}=1$ for later reference.

For $p \geq m$ :

$$
\tilde{\lambda}^{(j)}(w)=\tilde{\lambda}^{(j)}-w \sum_{s \in\left\{k^{+}\right\}} \tilde{\lambda}^{(s)}, \quad \lambda^{(k)}(w)=\lambda^{(k)}+w \lambda^{(j)}, \quad \forall k \in\left\{k^{+}\right\}
$$

and $j$ a negative helicity graviton.

If $m \geq p$ the deformation is

$$
\lambda^{(i)}(w)=\lambda^{(i)}+w \sum_{s \in\left\{r^{-}\right\}} \lambda^{(s)}, \quad \tilde{\lambda}^{(k)}(w)=\tilde{\lambda}^{(k)}-w \tilde{\lambda}^{(i)}, \quad \forall k \in\left\{r^{-}\right\}
$$

and $i$ a positive helicity graviton.

The proof that this choice gives $M_{n}(w) \rightarrow 0$ as $w \rightarrow \infty$ and more details are given in the appendix. The proof involves a careful analysis of when the $w$ can possibly drop out of propagators. This is basically the point where all other deformations fail.

Here we simply give the final form of the auxiliary recursion relations. Again we have to distinguish cases. If $p \geq m$ we write $M_{n}$ as sums of products of amplitudes with less than $n$ gravitons as follows:

$$
\begin{aligned}
& M_{n}\left(\left\{r^{-}\right\},\left\{k^{+}\right\}\right)= \\
& \quad=\sum_{\mathcal{I}} \sum_{h= \pm} M_{\mathcal{I}}\left(\left\{r_{\mathcal{I}}^{-}\right\},\left\{k_{\mathcal{I}}^{+}\left(w_{\mathcal{I}}\right)\right\},-P_{\mathcal{I}}^{h}\left(w_{\mathcal{I}}\right)\right) \frac{1}{P_{\mathcal{I}}^{2}} M_{\mathcal{J}}\left(\left\{r_{\mathcal{J}}^{-}\left(w_{\mathcal{I}}\right)\right\},\left\{k_{\mathcal{J}}^{+}\left(w_{\mathcal{I}}\right)\right\}, P_{\mathcal{I}}^{-h}\left(w_{\mathcal{I}}\right)\right)
\end{aligned}
$$


where:

- $\mathcal{I}$ and $\mathcal{J}$ are subsets of the set $\{1, \ldots, n\}$ such that $\mathcal{I} \cup \mathcal{J}=\{1, \ldots, n\}$. The sum is over all partitions $\{\mathcal{I}, \mathcal{J}\}$ of $\{1, \ldots, n\}$ such that at least one positive helicity graviton is in $\mathcal{I}$ and $j \in \mathcal{J}$.

- $P_{\mathcal{I}}$ is the sum of all the momenta of gravitons in $\mathcal{I}$;

- $\left\{r_{\mathcal{I}}^{-}\right\} \equiv \mathcal{I}^{-}$is the set of negative helicity gravitons in $\mathcal{I}$;

- $\left\{r_{\mathcal{J}}^{-}\left(w_{\mathcal{I}}\right)\right\}$ is the set of negative helicity gravitons in $\mathcal{J}$. The $w_{\mathcal{I}}$ dependence is only through $\tilde{\lambda}^{(j)}\left(w_{\mathcal{I}}\right)$;

- $\left\{k_{\mathcal{I}}^{+}\left(w_{\mathcal{I}}\right)\right\} \equiv \mathcal{I}^{+}$is the set of positive helicity gravitons in $\mathcal{I}$. All of them have been deformed and their dependence on $w_{\mathcal{I}}$ is only through

$$
\lambda^{(k)}\left(w_{\mathcal{I}}\right)=\lambda^{(k)}+w_{\mathcal{I}} \lambda^{(j)}
$$

- $\left\{k_{\mathcal{J}}^{+}\left(w_{\mathcal{I}}\right)\right\}$ is the set of positive helicity gravitons in $\mathcal{J}$. All of them have also been deformed via (3.10).

- The deformation parameter is given by

$$
w_{\mathcal{I}}=\frac{P_{\mathcal{I}}^{2}}{\sum_{k \in \mathcal{I}^{+}}\left\langle j\left|P_{\mathcal{I}}\right| k\right]} .
$$

This definition ensures that the momentum

$$
P_{\mathcal{I}}\left(w_{\mathcal{I}}\right)_{a \dot{a}}=P_{\mathcal{I} a \dot{a}}+w_{\mathcal{I}} \lambda_{a}^{(j)} \sum_{k \in \mathcal{I}^{+}} \tilde{\lambda}_{\dot{a}}^{(k)}
$$

is a null vector, i.e., $P_{\mathcal{I}}\left(w_{\mathcal{I}}\right)^{2}=0$.

Now, if $m \geq p$ then we write $M_{n}$ as a sum over terms involving the product of amplitudes with less than $n$ gravitons as follows:

$$
\begin{aligned}
& M_{n}\left(\left\{r^{-}\right\},\left\{k^{+}\right\}\right)= \\
& \quad=\sum_{\mathcal{I}} \sum_{h= \pm} M_{\mathcal{I}}\left(\left\{r_{\mathcal{I}}^{-}\left(w_{\mathcal{I}}\right)\right\},\left\{k_{\mathcal{I}}^{+}\left(w_{\mathcal{I}}\right)\right\},-P_{\mathcal{I}}^{h}\left(w_{\mathcal{I}}\right)\right) \frac{1}{P_{\mathcal{I}}^{2}} M_{\mathcal{J}}\left(\left\{r_{\mathcal{J}}^{-}\left(w_{\mathcal{I}}\right)\right\},\left\{k_{\mathcal{J}}^{+}\right\}, P_{\mathcal{I}}^{-h}\left(w_{\mathcal{I}}\right)\right)
\end{aligned}
$$

where most definitions are as in the $p \geq m$ case except that the sets $\mathcal{I}$ and $\mathcal{J}$ are such that $i \in \mathcal{I}$ and all the negative helicity gravitons and the $i^{\text {th }}$ positive helicity graviton are deformed via (3.8) instead of (3.7).

The two rules, (3.9) and (3.13), provide a full set of recursion relations for gravity amplitudes. To see this note that using them one can express any $n$-graviton amplitude as the sum of products of two amplitudes with less than $n$ gravitons. The smaller amplitudes which depend on deformed spinors and the intermediate null vector $P\left(w_{\mathcal{I}}\right)$ are completely "physical" in the sense that by construction their momenta are on-shell and satisfy momentum conservation. Therefore they admit a definition in terms of Feynman diagrams again and can serve as a starting point to apply either (3.9) or (3.13), depending on the new number of plus and minus helicity gravitons. 


\subsection{Induction And Feynman Diagram Argument}

Consider any $n$-graviton amplitude under the BCFW deformation (2.1) on gravitons $i^{+}$ and $j^{-}$:

$$
\lambda^{(i)}(z)=\lambda^{(i)}+z \lambda^{(j)}, \quad \tilde{\lambda}^{(j)}(z)=\tilde{\lambda}^{(j)}-z \tilde{\lambda}^{(i)} .
$$

Without loss of generality we can assume that $M_{n}$ has $p \geq m$ and use (3.9) as our starting point. If $m \geq p$ we use (3.13) and everything that follows applies equally well.

Note that the choice of deformed gravitons in (3.14) is correlated to that in (3.9) or (3.13).

Our goal now is to prove that by using (3.14) on (3.9) the function $M_{n}(z)$ vanishes as $z$ is taken to infinity.

Let us consider each term in the sum of (3.9) individually. There are two classes of terms. The first kind is when $\{i, j\} \subset \mathcal{J}$. The second kind is when $i \in \mathcal{I}$ and $j \in \mathcal{J}$.

Consider a term of the first kind,

$$
\sum_{h= \pm} M_{\mathcal{I}}\left(\left\{r_{\mathcal{I}}^{-}\right\},\left\{k_{\mathcal{I}}^{+}\left(w_{\mathcal{I}}\right)\right\},-P_{\mathcal{I}}^{h}\left(w_{\mathcal{I}}\right)\right) \frac{1}{P_{\mathcal{I}}^{2}} M_{\mathcal{J}}\left(\left\{r_{\mathcal{J}}^{-}\left(w_{\mathcal{I}}, z\right)\right\},\left\{k_{\mathcal{J}}^{+}\left(w_{\mathcal{I}}, z\right)\right\}, P_{\mathcal{I}}^{-h}\left(w_{\mathcal{I}}\right)\right)
$$

Since both $i^{+}$and $j^{-}$belong to $\mathcal{J}$, the momentum $P_{\mathcal{I}}$ does not depend on $z$. Likewise from the definition of $w_{\mathcal{I}}$ in (3.11) one can see that it does not depend on $z$. Therefore, the $z$ dependence is confined to the second amplitude in (3.15) which we can write more explicitly as

$$
M_{\mathcal{J}}\left(\left\{r_{\mathcal{J}^{\prime}}^{-}\right\},\left\{k_{\mathcal{J}^{\prime}}^{+}\left(w_{\mathcal{I}}\right)\right\},\left\{\lambda^{(i)}\left(w_{\mathcal{I}}, z\right), \tilde{\lambda}^{(i)}\right\},\left\{\lambda^{(j)}, \tilde{\lambda}^{(j)}\left(w_{\mathcal{I}}, z\right)\right\}, P_{\mathcal{I}}^{-h}\left(w_{\mathcal{I}}\right)\right)
$$

where the set $\mathcal{J}^{\prime}=\mathcal{J} \backslash\{i, j\}$. It is straightforward to show that

$$
\lambda^{(i)}\left(w_{\mathcal{I}}, z\right)=\lambda^{(i)}\left(w_{\mathcal{I}}\right)+z \lambda^{(j)}, \quad \tilde{\lambda}^{(j)}\left(w_{\mathcal{I}}, z\right)=\tilde{\lambda}^{(j)}\left(w_{\mathcal{I}}\right)-z \tilde{\lambda}^{(i)} .
$$

The fact that $\lambda^{(i)}\left(w_{\mathcal{I}}\right)$ and $\tilde{\lambda}^{(j)}\left(w_{\mathcal{I}}\right)$ get deformed exactly in the same way as $\lambda^{(i)}$ and $\tilde{\lambda}^{(j)}$ do is what allows us to use induction for these terms. Note that the amplitude (3.16) is therefore a physical amplitude with a BCFW deformation. The number of gravitons is less than $n$ and by our induction hypothesis it vanishes as $z$ goes to infinity.

To complete the induction argument it suffices to note that the auxiliary recursion relations we are using can reduce any amplitude to products of three graviton amplitudes. Finally, recall that the Feynman diagram argument at the beginning of this section showed that amplitudes with less than five gravitons vanish at infinity under the BCFW deformation.

Consider now a term of the second kind,

$$
\begin{aligned}
\sum_{h= \pm} & M_{\mathcal{I}}\left(\left\{r_{\mathcal{I}}^{-}\right\},\left\{k_{\mathcal{I}}^{+}\left(w_{\mathcal{I}}(z), z\right)\right\},-P_{\mathcal{I}}^{h}\left(w_{\mathcal{I}}(z), z\right)\right) \frac{1}{P_{\mathcal{I}}^{2}(z)} \\
& M_{\mathcal{J}}\left(\left\{r_{\mathcal{J}}^{-}\left(w_{\mathcal{I}}(z), z\right)\right\},\left\{k_{\mathcal{J}}^{+}\left(w_{\mathcal{I}}(z)\right)\right\}, P_{\mathcal{I}}^{-h}\left(w_{\mathcal{I}}(z), z\right)\right) .
\end{aligned}
$$


Recall that for these terms $i^{+} \in \mathcal{I}$ while $j^{-} \in \mathcal{J}$. The $z$ dependence we have displayed in (3.18) looks complicated at first since

$$
w_{\mathcal{I}}(z)=\frac{P_{\mathcal{I}}(z)^{2}}{\sum_{k \in \mathcal{I}^{+}}\left\langle j\left|P_{\mathcal{I}}(z)\right| k\right]}
$$

appears to be a rational function of $z$ since $P_{\mathcal{I}}(z)_{a \dot{a}}=P_{\mathcal{I} a \dot{a}}+z \lambda_{a}^{(j)} \tilde{\lambda}_{\dot{a}}^{(i)}$. Note, however, that $\tilde{\lambda}^{(k)}$ 's with $k \in \mathcal{I}^{+}$do not depend on $z$ and that the $z$ dependence $z \lambda_{a}^{(j)} \tilde{\lambda}_{\dot{a}}^{(i)}$ in $P_{\mathcal{I}}(z)$ drops out of the denominator thanks to the contraction with $\langle j|$.

Then we find that $w_{\mathcal{I}}(z)$ is simply a linear function of $z$ :

$$
w_{\mathcal{I}}(z)=w_{\mathcal{I}}-z\left(\frac{\left\langle j\left|P_{\mathcal{I}}\right| i\right]}{\sum_{k \in \mathcal{I}^{+}}\left\langle j\left|P_{\mathcal{I}}\right| k\right]}\right)
$$

where $w_{\mathcal{I}}$ is just the undeformed one, i.e., $w_{\mathcal{I}}(0)$.

The final step before we proceed to study the behavior for $z \rightarrow \infty$ using Feynman diagrams is to determine the properties of the internal graviton that enters with opposite helicities in the amplitudes of (3.18). The momentum of the internal graviton is given by

$$
P_{\mathcal{I}}\left(w_{\mathcal{I}}(z), z\right)=\sum_{k \in \mathcal{I}^{-}} p_{k}+p_{i}\left(w_{\mathcal{I}}(z), z\right)+\sum_{s \in \mathcal{I}^{+}, s \neq i} p_{s}\left(w_{\mathcal{I}}(z)\right) .
$$

The important observation is that the $z$-dependence can be fully separated as follows

$$
P_{\mathcal{I}}\left(w_{\mathcal{I}}(z), z\right)=P_{\mathcal{I}}\left(w_{\mathcal{I}}\right)+z \lambda^{(j)}\left(-\left(\frac{\left\langle j\left|P_{\mathcal{I}}\right| i\right]}{\sum_{k \in \mathcal{I}^{+}}\left\langle j\left|P_{\mathcal{I}}\right| k\right]}\right) \sum_{s \in \mathcal{I}^{+}} \tilde{\lambda}^{(s)}+\tilde{\lambda}^{(i)}\right)
$$

where $P_{\mathcal{I}}\left(w_{\mathcal{I}}\right)$ is the $z$-undeformed one, i.e., $P_{\mathcal{I}}\left(w_{\mathcal{I}}(0), 0\right)$.

Note that we have written $P_{\mathcal{I}}\left(w_{\mathcal{I}}(z), z\right)$, which is a null vector, as the sum of two null vectors. For real momenta, this would imply that all three vectors are proportional. However, in this case all three vectors are complex and all that is required is that either all $\lambda$ 's or all $\tilde{\lambda}$ 's be proportional. We claim that in this particular case all $\tilde{\lambda}$ 's are proportional. To see this note that if we write $P_{\mathcal{I}}\left(w_{\mathcal{I}}\right)_{a \dot{a}}=\lambda_{a}^{(P)} \tilde{\lambda}_{\dot{a}}^{(P)}$, then $\tilde{\lambda}_{\dot{a}}^{(P)}$ is proportional to $\zeta_{\dot{a}}=$ $\eta^{a} P_{\mathcal{I}}\left(w_{\mathcal{I}}\right)_{a \dot{a}}$ for some arbitrary spinor $\eta^{a}$.

We claim that the $\tilde{\lambda}$ spinor of the vector multiplying $z$ in (3.22) is also proportional to $\zeta^{\dot{a}}$ if $\eta_{a}=\lambda_{a}^{(j)}$. In this case, $\zeta_{\dot{a}}=\lambda^{(j) a} P_{\mathcal{I}}\left(w_{\mathcal{I}}\right)_{a \dot{a}}=\lambda^{(j) a} P_{\mathcal{I} a \dot{a}}$. To prove our claim consider the inner product of the two spinors

$$
\left(-\left(\frac{\left\langle j\left|P_{\mathcal{I}}\right| i\right]}{\sum_{k \in \mathcal{I}^{+}}\left\langle j\left|P_{\mathcal{I}}\right| k\right]}\right) \sum_{s \in \mathcal{I}^{+}} \tilde{\lambda}_{\dot{a}}^{(s)}+\tilde{\lambda}_{\dot{a}}^{(i)}\right) \zeta^{\dot{a}}=\left(\left(\frac{\left\langle j\left|P_{\mathcal{I}}\right| i\right]}{\sum_{k \in \mathcal{I}^{+}}\left\langle j\left|P_{\mathcal{I}}\right| k\right]}\right) \sum_{s \in \mathcal{I}^{+}}\left\langle j\left|P_{\mathcal{I}}\right| s\right]-\left\langle j\left|P_{\mathcal{I}}\right| i\right]\right) .
$$

The right hand side of (3.23) vanishes trivially showing that the two spinors are proportional.

Therefore, it follows that we can write $P_{\mathcal{I}}\left(w_{\mathcal{I}}(z), z\right)_{a \dot{a}}=\lambda_{a}(z) \tilde{\lambda}_{\dot{a}}^{P}$ where $\lambda_{a}(z)=\lambda_{a}^{(P)}+$ $z \beta \lambda_{a}^{(j)}$ for some $\beta$ which is $z$ independent. Note that if $z=0$ we recover $P_{\mathcal{I}}\left(w_{\mathcal{I}}\right)_{a \dot{a}}=$ $\lambda_{a}^{(P)} \tilde{\lambda}_{\dot{a}}^{(P)}$. 
Let us turn to the analysis of the amplitudes in (3.18) to show that their product vanishes as $z$ is taken to infinity. In other words, we will see that $M_{\mathcal{I}}$ and $M_{\mathcal{J}}$ may not vanish simultaneously but their product together with the propagator always does.

Consider the first amplitude $M_{\mathcal{I}}\left(\left\{r_{\mathcal{I}}^{-}\right\},\left\{k_{\mathcal{I}}^{+}\left(w_{\mathcal{I}}(z), z\right)\right\},-P_{\mathcal{I}}^{h}\left(w_{\mathcal{I}}(z), z\right)\right)$. Let the number of particles in the sets $\left\{r_{\mathcal{I}}^{-}\right\}$and $\left\{k_{\mathcal{I}}^{+}\right\}$be $m_{\mathcal{I}}$ and $p_{\mathcal{I}}$ respectively ${ }^{7}$.

The Feynman diagram analysis is very similar to that performed at the beginning of section III. The leading Feynman diagram is again one with only cubic vertices that posses a quadratic dependence on momenta. The number of cubic vertices is the total number of particles ${ }^{8}$ minus two, i.e, $m_{\mathcal{I}}+p_{\mathcal{I}}-1$. Therefore the contribution from vertices gives at most a factor of $z^{2\left(m_{\mathcal{I}}+p_{\mathcal{I}}-1\right)}$. There are $p_{\mathcal{I}}+1$ polarization vectors that depend on $z$, giving a total contribution of $1 / z^{2\left(p_{\mathcal{I}}+h\right)}$. Here we have used that since $z$ enters in $-P_{\mathcal{I}}^{h}\left(w_{\mathcal{I}}(z), z\right)$ only through $\lambda(z)$, its polarization tensor gives a contribution of $1 / z^{2 h}$. Finally, we need to count the number of propagators that depend on $z$. It turns out that there are exactly $m_{\mathcal{I}}+p_{\mathcal{I}}-2$ of them giving a contribution of $1 / z^{m_{\mathcal{I}}+p_{\mathcal{I}}-2}$. This last statement is not obvious since there could be accidental cancelations of the $z$ dependence. Let us continue with the argument here and we will prove that there is no accidental cancelations within the propagators in the next subsection ${ }^{9}$. Collecting all factors we get

$$
M_{\mathcal{I}}\left(\left\{r_{\mathcal{I}}^{-}\right\},\left\{k_{\mathcal{I}}^{+}\left(w_{\mathcal{I}}(z), z\right)\right\},-P_{\mathcal{I}}^{h}\left(w_{\mathcal{I}}(z), z\right)\right) \sim \frac{1}{z^{p_{\mathcal{I}}-m_{\mathcal{I}}+2 h}} .
$$

The propagator $1 / P_{\mathcal{I}}^{2}(z)$ in $(3.18)$ goes as $1 / z$.

The reader might have noticed that in this argument special care is required when $\mathcal{I}^{+}=\{i\}$. We postpone the study of this case to the end of the section. Until then we simply assume that $i \in \mathcal{I}^{+}$but $\mathcal{I}^{+} \neq\{i\}$.

Consider now the second amplitude in (3.18),

$$
M_{\mathcal{J}}\left(\left\{r_{\mathcal{J}}^{-}\left(w_{\mathcal{I}}(z), z\right)\right\},\left\{k_{\mathcal{J}}^{+}\left(w_{\mathcal{I}}(z)\right)\right\}, P_{\mathcal{I}}^{-h}\left(w_{\mathcal{I}}(z), z\right)\right)
$$

Let the number of gravitons in $\left\{r_{\mathcal{J}}^{-}\right\}$and $\left\{k_{\mathcal{J}}^{+}\right\}$be $m_{\mathcal{J}}$ and $p_{\mathcal{J}}$ respectively.

The cubic vertices give again a factor of $z^{2\left(p_{\mathcal{J}}+m_{\mathcal{J}}-1\right)}$. The polarization tensors give a factor of $1 / z^{2\left(p_{\mathcal{J}}-h+1\right)}$. Here we have taken into account the contribution from the $z$ dependent negative helicity graviton, i.e, the $j^{\text {th }}$ graviton, and from the internal graviton, $P_{\mathcal{I}}^{-h}\left(w_{\mathcal{I}}(z), z\right)$. Finally, the propagators contribute again a factor of $1 / z^{p_{\mathcal{J}}+m_{\mathcal{J}}-2}$. Collecting all factors we get

$$
M_{\mathcal{J}}\left(\left\{r_{\mathcal{J}}^{-}\left(w_{\mathcal{I}}(z), z\right)\right\},\left\{k_{\mathcal{J}}^{+}\left(w_{\mathcal{I}}(z)\right)\right\}, P_{\mathcal{I}}^{-h}\left(w_{\mathcal{I}}(z), z\right)\right) \sim \frac{1}{z^{p_{\mathcal{J}}-m_{\mathcal{J}}-2(h-1)}} .
$$

Combining all contributions from (3.24), the propagator and (3.26), the leading $z$ behavior of $(3.18)$ is $1 / z^{p-m+3}$.

\footnotetext{
${ }^{7}$ Note that if $h=+$ this is a physical amplitude where only the $\lambda$ 's of positive helicity gravitons have been deformed. It is interesting to note that this deformation is basically the one introduced by Risager in [35] and later in [34] to construct an MHV diagram expansion for gravity amplitudes.

${ }^{8}$ The total number of gravitons in $M_{\mathcal{I}}$ is $m_{\mathcal{I}}+p_{\mathcal{I}}+1$ since $-P_{\mathcal{I}}^{h}\left(w_{\mathcal{I}}(z), z\right)$ should also be included.

${ }^{9}$ More precisely, what we prove in the next subsection is that trivial cancelations in which neither propagators nor vertices depend on $z$ are the only ones that can occur.
} 
This shows that all the amplitudes with $p \geq m$ vanish at infinity.

As stated at the beginning of this subsection, a similar discussion holds for the case of amplitudes with $m \geq p$ : by repeating the same counting starting from relation (3.13), the behavior at infinity of terms of the second kind turns out to be $1 / z^{m-p+3}$. Terms of the first kind can again be treated by induction.

It is important to mention that the way amplitudes vanish at infinity is generically only as $1 / z^{2}$. This is because terms of the first kind which are treated by induction vanish as three-graviton amplitudes do, i.e, as $1 / z^{2}$.

This completes our proof of the vanishing of $M_{n}(z)$ as $z$ goes to infinity up to the claim made about the number of propagators that contribute a $1 / z$ factor and the exceptional case when $\mathcal{I}^{+}=\{i\}$. We now turn to these crucial steps of our proof.

\subsection{Analysis Of The Contribution From Propagators}

One thing left to prove is that in the leading Feynman diagrams contributing to the first amplitude, $M_{\mathcal{I}}$, there are exactly $m_{\mathcal{I}}+p_{\mathcal{I}}-2$ propagators giving a $1 / z$ contribution at infinity while in the second amplitude, $M_{\mathcal{J}}$, there are exactly $m_{\mathcal{J}}+p_{\mathcal{J}}-2$ of them.

\subsubsection{Propagators In Leading Feynman Diagrams Of $M_{\mathcal{I}}$}

Let us start with $M_{\mathcal{I}}$. The argument here uses similar elements to the ones given in the appendix where we provided a proof of the auxiliary recursion relations.

Consider a given Feynman diagram. A propagator naturally divides the diagram into two subdiagrams. Let use denote them by $\mathcal{L}$ and $\mathcal{R}$. Without loss of generality, we can always take the graviton with momentum $-P_{\mathcal{I}}^{h}\left(w_{\mathcal{I}}(z), z\right)$ to be in $\mathcal{R}$. In the set of positive helicity gravitons, $\left\{k_{\mathcal{I}}^{+}\left(w_{\mathcal{I}}(z), z\right)\right\}$, there is one that is special; the $i^{\text {th }}$ graviton. We consider two cases, the first is when $i \in \mathcal{L}^{+}$and the second when $i \in \mathcal{R}^{+}$.

Case $A: i \in \mathcal{L}^{+}$

Let $i \in \mathcal{L}^{+}$, then the propagator under consideration has the form

$$
P_{\mathcal{L}}\left(w_{\mathcal{I}}(z), z\right)=P_{\mathcal{L}}\left(w_{\mathcal{I}}(0)\right)+z \lambda^{(j)}\left(-\frac{\left\langle j\left|P_{\mathcal{I}}\right| i\right]}{\sum_{k \in \mathcal{I}^{+}}\left\langle j\left|P_{\mathcal{I}}\right| k\right]} \sum_{s \in \mathcal{L}^{+}} \tilde{\lambda}^{(s)}+\tilde{\lambda}^{(i)}\right) .
$$

We are interested in asking when

$$
P_{\mathcal{L}}\left(w_{\mathcal{I}}(z), z\right)^{2}=P_{\mathcal{L}}\left(w_{\mathcal{I}}(0)\right)^{2}+z\left(\frac{\left\langle j\left|P_{\mathcal{I}}\right| i\right] \sum_{k \in \mathcal{L}^{+}}\left\langle j\left|P_{\mathcal{L}}\right| k\right]}{\sum_{k \in \mathcal{I}^{+}}\left\langle j\left|P_{\mathcal{I}}\right| k\right]}-\left\langle j\left|P_{\mathcal{L}}\right| i\right]\right)
$$

can be $z$ independent. Therefore we have to analyze under which conditions the factor multiplying $z$ can be zero for a generic choice of momenta and polarization tensors of the physical gravitons subject only to the overall momentum conservation constrain.

Let us write the factor of interest as follows

$$
\left\langle j\left|P_{\mathcal{I}}\right| i\right] \sum_{k \in \mathcal{L}^{+}}\left\langle j\left|P_{\mathcal{L}}\right| k\right]-\left\langle j\left|P_{\mathcal{L}}\right| i\right] \sum_{k \in \mathcal{I}^{+}}\left\langle j\left|P_{\mathcal{I}}\right| k\right]=\lambda^{(j) a} \lambda^{(j) b} P_{\mathcal{L} a \dot{a}} P_{\mathcal{I} b \dot{b}} T^{\dot{a} \dot{b}}
$$


with

$$
T^{\dot{a} \dot{b}}=\tilde{\lambda}^{(i) \dot{a}} \sum_{k \in \mathcal{L}^{+}} \tilde{\lambda}^{(k) \dot{b}}-\tilde{\lambda}^{(i) \dot{b}} \sum_{k \in \mathcal{I}^{+}} \tilde{\lambda}^{(k) \dot{a}} .
$$

Here we have to consider two different cases $^{10}$ :

- $\mathcal{I}^{+} \backslash \mathcal{L}^{+} \neq \emptyset$.

- $\mathcal{I}^{+}=\mathcal{L}^{+}$and $\mathcal{L}^{+} \neq\{i\}$.

Let us start by assuming that $\mathcal{I}^{+} \backslash \mathcal{L}^{+}$is non-empty and that, say, $s \in \mathcal{I}^{+} \backslash \mathcal{L}^{+}$. The space of kinematical invariants we consider is determined by the momentum and polarization tensors of each of the original gravitons. Consider both objects for the $s^{\text {th }}$ graviton

$$
\epsilon_{a \dot{a}, b \dot{b}}^{+(s)}=\frac{\mu_{a} \tilde{\lambda}_{\dot{a}}^{(s)} \mu_{b} \tilde{\lambda}_{\dot{b}}^{(s)}}{\left\langle\mu, \lambda^{(s)}\right\rangle^{2}}, \quad p_{a \dot{a}}^{(s)}=\lambda_{a}^{(s)} \tilde{\lambda}_{\dot{a}}^{(s)}
$$

It is clear that if we take $\left\{\lambda_{a}^{(s)}, \tilde{\lambda}_{\dot{a}}^{(s)}\right\}$ to $\left\{t^{-1} \lambda_{a}^{(s)}, t \tilde{\lambda}_{\dot{a}}^{(s)}\right\}$ with $t$ a fourth root of unity, i.e, $t^{4}=1$ then (3.31) is invariant. Therefore, any quantity that vanishes for $t=1$ must also vanish for all four values of $t$. In particular, it must be the case that (3.29) must vanish for all four values of $t$. Since momentum is not affected only the tensor $T^{\dot{a} \dot{b}}$ changes. Taking the difference between two values of $t$, say $t=1$ and $t=i$, we find that $\left.T^{\dot{a} \dot{b}}\right|_{t=1}-\left.T^{\dot{a} \dot{b}}\right|_{t=i} \sim \tilde{\lambda}^{(i)} \dot{b} \tilde{\lambda}^{(s) \dot{a}}$. Therefore, the vanishing of (3.29) implies that of

$$
\left\langle j\left|P_{\mathcal{L}}\right| i\right]\left\langle j\left|P_{\mathcal{I}}\right| s\right]=0 .
$$

This condition is then equivalent to

$$
\operatorname{tr}\left(\not p_{j} \not P_{\mathcal{L}} \not p_{i} \not P_{\mathcal{L}}\right)=0 \quad \text { or } \quad \operatorname{tr}\left(\not p_{j} \not P_{\mathcal{I}} \not p_{s} \not P_{\mathcal{I}}\right)=0
$$

but these are constraints on the kinematical space which are not satisfied at generic points.

The second case we have to consider is when $\mathcal{I}^{+}=\mathcal{L}^{+}$and $\mathcal{L}^{+} \neq\{i\}$. Let us introduce the notation $\tilde{\mu}_{\dot{a}}=\sum_{k \in \mathcal{I}^{+}} \tilde{\lambda}_{\dot{a}}^{(k)}$. Therefore the condition we want to exclude is

$$
\left\langle j\left|P_{\mathcal{I}}\right| i\right]\left\langle j\left|P_{\mathcal{L}}\right| \tilde{\mu}\right]-\left\langle j\left|P_{\mathcal{L}}\right| i\right]\left\langle j\left|P_{\mathcal{I}}\right| \tilde{\mu}\right]=0 .
$$

Using Schouten's identity we can write this as

$$
\left\langle j\left|P_{\mathcal{I}} P_{\mathcal{L}}\right| j\right\rangle[i, \tilde{\mu}]=0 .
$$

The vanishing of either factor ${ }^{11}$ implies a constraint for the space of kinematical invariants. In the case of the second factor this can easily be seen by choosing $s \in \mathcal{I}^{+}$and $s \neq i$, then using the scaling by $t$ with $t^{4}=1$ to conclude that $\left(p_{s}+p_{i}\right)^{2}=0$.

This completes the proof that the $z$ dependence cannot drop out of any propagator and therefore all $m_{\mathcal{I}}+p_{\mathcal{I}}-2$ of them give a $1 / z$ factor in $M_{\mathcal{I}}$ if $i \in \mathcal{L}$.

\footnotetext{
${ }^{10}$ There are actually three cases. The third is when $\mathcal{I}^{+}=\mathcal{L}^{+}=\{i\}$ but this is part of the special case that is considered at then end of the section.

${ }^{11}$ See section 1.A for the explanation of the notation in the first factor.
} 


\section{Case B: $i \in \mathcal{R}^{+}$}

The analysis when $i \in \mathcal{R}$ is completely analogous except for the fact that there is one case that was not possible before. As we will show, this will correspond to diagrams which give a non-leading contribution.

Consider the analog of (3.28)

$$
P_{\mathcal{L}}\left(w_{\mathcal{I}}(z), z\right)^{2}=P_{\mathcal{L}}\left(w_{\mathcal{I}}(0)\right)^{2}+z\left\langle j\left|P_{\mathcal{I}}\right| i\right]\left(\frac{\sum_{k \in \mathcal{L}^{+}}\left\langle j\left|P_{\mathcal{L}}\right| k\right]}{\sum_{k \in \mathcal{I}^{+}}\left\langle j\left|P_{\mathcal{I}}\right| k\right]}\right) .
$$

The new case is when $\mathcal{L}^{+}=\emptyset$, then the $z$ dependence drops out. Of course, this is not a problem because if the set $\mathcal{L}^{+}$is empty it means that nothing on the subdiagram $\mathcal{L}$ depends on $z$, including the cubic vertices. Therefore, neither propagators nor cubic vertices contribute. One can then concentrate on the subdiagram $\mathcal{R}$, but this subdiagram has less particles than the total diagram and the same number of $z$-dependent polarization tensors. Therefore these diagrams go to zero even faster than diagrams where $\mathcal{L}^{+}$is not empty.

\subsubsection{Propagators In Leading Feynman Diagrams Of $M_{\mathcal{J}}$}

Let us now study the leading Feynman diagrams contributing to $M_{\mathcal{J}}$. Again, the propagator divides the diagram in two subdiagrams that we denote $\mathcal{L}$ and $\mathcal{R}$. Without loss of generality, we can always take the graviton with momentum $P_{\mathcal{I}}^{-h}\left(w_{\mathcal{I}}(z), z\right)$ to be in $\mathcal{R}$. As in the previous discussion we have a special graviton, i.e, the $j^{\text {th }}$ graviton. Therefore we have to consider two cases, $j \in \mathcal{L}$ and $j \in \mathcal{R}$.

Case $A: j \in \mathcal{L}^{-}$

Let us first consider the case $j \in \mathcal{L}$. The $z$ dependence of $\tilde{\lambda}^{(j)}\left(w_{\mathcal{I}}(z), z\right)$ is the most complicated of all. This is why we write it explicitly

$$
\tilde{\lambda}^{(j)}\left(w_{\mathcal{I}}(z), z\right)_{\dot{a}}=\tilde{\lambda}^{(j)}\left(w_{\mathcal{I}}\right)_{\dot{a}}+z\left(-\tilde{\lambda}_{\dot{a}}^{(i)}+\frac{\left\langle j\left|P_{\mathcal{I}}\right| i\right]}{\sum_{k \in \mathcal{I}^{+}}\left\langle j\left|P_{\mathcal{I}}\right| k\right]} \sum_{s \in\left\{k^{+}\right\}} \tilde{\lambda}_{\dot{a}}^{(s)}\right) .
$$

Using this and the fact that the set of labels of all positive helicity gravitons $\left\{k^{+}\right\}$must be equal to $\mathcal{I}^{+} \cup \mathcal{J}^{+}$, we find that the propagator of interest has a momentum dependence of the form

$$
P_{\mathcal{L}}\left(w_{\mathcal{I}}(z), z\right)^{2}=P_{\mathcal{L}}\left(w_{\mathcal{I}}(0)\right)^{2}+z\left(\left\langle j\left|P_{\mathcal{L}}\right| i\right]-\left\langle j\left|P_{\mathcal{I}}\right| i\right] \frac{\sum_{k \in \mathcal{I}^{+} \cup\left(\mathcal{J}^{+} \backslash \mathcal{L}^{+}\right)}\left\langle j\left|P_{\mathcal{L}}\right| k\right]}{\sum_{k \in \mathcal{I}^{+}}\left\langle j\left|P_{\mathcal{I}}\right| k\right]}\right) .
$$

We are then interested in asking when this expression can be $z$ independent.

The analysis is similar to the one given for $M_{\mathcal{I}}$ so we will be brief. The factor of interest is now

$$
\left\langle j\left|P_{\mathcal{L}}\right| i\right] \sum_{k \in \mathcal{I}^{+}}\left\langle j\left|P_{\mathcal{I}}\right| k\right]-\left\langle j\left|P_{\mathcal{I}}\right| i\right] \sum_{k \in \mathcal{I}^{+} \cup\left(\mathcal{J}^{+} \backslash \mathcal{L}^{+}\right)}\left\langle j\left|P_{\mathcal{L}}\right| k\right] .
$$

We have to consider two cases: 
- $\mathcal{J}^{+} \backslash \mathcal{L}^{+} \neq \emptyset$.

- $\mathcal{J}^{+}=\mathcal{L}^{+}$and $\mathcal{I}^{+} \neq\{i\}$.

In the first case we can assume that, say, the $s^{\text {th }}$ graviton is in $\mathcal{J}^{+} \backslash \mathcal{L}^{+}$. Then by using the argument that any statement about $\left\{\lambda^{(s)}, \tilde{\lambda}^{(s)}\right\}$ must also be true for $\left\{t^{-1} \lambda^{(s)}, t \tilde{\lambda}^{(s)}\right\}$ with $t^{4}=1$ one can show that the vanishing of (3.39) implies a nontrivial constraint on kinematical invariants that is not generically satisfied.

The second case is also similar to one we considered in the analysis of $M_{\mathcal{I}}$. Here we have that $\mathcal{I}^{+} \cup\left(\mathcal{J}^{+} \backslash \mathcal{L}^{+}\right)=\mathcal{I}^{+} \cup \emptyset=\mathcal{I}^{+}$. Therefore (3.39) becomes

$$
\left\langle j\left|P_{\mathcal{L}}\right| i\right]\left\langle j\left|P_{\mathcal{I}}\right| \tilde{\mu}\right]-\left\langle j\left|P_{\mathcal{I}}\right| i\right]\left\langle j\left|P_{\mathcal{L}}\right| \tilde{\mu}\right]
$$

where $\tilde{\mu}_{\dot{a}}=\sum_{s \in \mathcal{I}^{+} \backslash\{i\}} \tilde{\lambda}^{(s)}$. Since by assumption $\mathcal{I}^{+} \backslash\{i\} \neq \emptyset$ we can use Schouten's identity to derive non-trivial constraints on the kinematical invariants which are not satisfied for generic momenta.

Recall that the case when $\mathcal{I}^{+}=\{i\}$ is special and will be treated separately.

Case $B: j \in \mathcal{R}^{-}$

In this case, the propagator of interest can be written as

$$
P_{\mathcal{L}}\left(w_{\mathcal{I}}(z), z\right)^{2}=P_{\mathcal{L}}\left(w_{\mathcal{I}}(0)\right)^{2}+z\left\langle j\left|P_{\mathcal{I}}\right| i\right]\left(\frac{\sum_{k \in \mathcal{L}^{+}}\left\langle j\left|P_{\mathcal{L}}\right| k\right]}{\sum_{k \in \mathcal{I}^{+}}\left\langle j\left|P_{\mathcal{I}}\right| k\right]}\right) .
$$

This is again similar to the corresponding case in $M_{\mathcal{I}}$. The only new case compared to when $j \in \mathcal{L}^{-}$is when $\mathcal{L}^{+}$is empty. Then nothing in $\mathcal{L}$ depends on $z$ and we can consider a Feynman diagram that has less minus helicity gravitons than the original one and therefore it goes faster to zero at infinity than the leading diagrams obtained when $\mathcal{L}^{+} \neq \emptyset$.

This conclude our discussion about the contribution of the propagators.

\subsection{Analysis Of The Special Case $\mathcal{I}^{+}=\{i\}$}

Let us now consider the final case. This is when $\mathcal{I}^{+}=\mathcal{L}^{+}=\{i\}$. This case is quite interesting since several unexpected cancelations take place. Consider $w_{\mathcal{I}}(z)$ given in $(3.20)$. In this case, it is easy to check that $w_{\mathcal{I}}(z)=w_{\mathcal{I}}(0)-z$. A consequence of this is that $\lambda^{(i)}\left(w_{\mathcal{I}}(z), z\right)=\lambda^{(i)}(z)+w_{\mathcal{I}}(z) \lambda^{(j)}$ becomes $z$-independent. To see this recall that $\lambda^{(i)}(z)=$ $\lambda^{(i)}+z \lambda^{(j)}$. Therefore $\lambda^{(i)}\left(w_{\mathcal{I}}(z), z\right)=\lambda^{(i)}\left(w_{\mathcal{I}}\right)$. This also implies that $P_{\mathcal{I}}^{h}\left(w_{\mathcal{I}}(z), z\right)$ is $z$ independent. Therefore, the full amplitude $M_{\mathcal{I}}$ is $z$ independent.

Recall that we are interested in the behavior of

$$
\sum_{h= \pm} M_{\mathcal{I}}^{h} \frac{1}{P_{\mathcal{I}}^{2}(z)} M_{\mathcal{J}}^{-h}(z)
$$

The propagator $1 / P_{\mathcal{I}}^{2}(z)$ contributes a factor of $1 / z$.

Now we have to look at

$$
M_{\mathcal{J}}(z)=M_{\mathcal{J}}\left(\left\{r_{\mathcal{J}}^{-}\left(w_{\mathcal{I}}(z), z\right)\right\},\left\{k_{\mathcal{J}}^{+}\left(w_{\mathcal{I}}(z)\right)\right\}, P_{\mathcal{I}}^{-h}\left(w_{\mathcal{I}}(z), z\right)\right) .
$$


Let us study the $z$ dependence of each graviton carefully. We have that the $j^{\text {th }}$ graviton (which has negative helicity) and all positive helicity gravitons in $\mathcal{J}^{+}=\left\{k_{\mathcal{J}}^{+}\left(w_{\mathcal{I}}(z)\right)\right\}$ behave as

$$
\tilde{\lambda}^{(j)}\left(w_{\mathcal{I}}(z), z\right)=\tilde{\lambda}^{(j)}\left(w_{\mathcal{I}}\right)+z \sum_{s \in \mathcal{J}^{+}} \tilde{\lambda}^{(s)}, \quad \lambda^{(s)}\left(w_{\mathcal{I}}(z)\right)=\lambda^{(s)}\left(w_{\mathcal{I}}\right)-z \lambda^{(j)} \quad \forall s \in \mathcal{J}^{+} .
$$

Close inspection of (3.43) shows a striking fact. This deformation is exactly the same as the one that led to the auxiliary recursion relations in the first place, i.e, the deformation given in (3.7) but using $z$ instead of $w$ as deformation parameter and $\tilde{\lambda}^{(j)}\left(w_{\mathcal{I}}\right)$ and $\lambda^{(s)}\left(w_{\mathcal{I}}\right)$ as undeformed spinors. Finally, recall that $P_{\mathcal{I}}\left(w_{\mathcal{I}}(z), z\right)$, which also appears in $M_{\mathcal{J}}$, was shown to be $z$ independent.

Now, if $h=+$ we have $P_{\mathcal{I}}^{-}\left(w_{\mathcal{I}}\right)$ and therefore, $M_{\mathcal{J}}(z)$ is nothing but a physical amplitude under the maximal deformation (3.7). In the appendix, we showed that amplitudes vanish as the deformation parameter, which in this case is $z$, is taken to infinity if the number of pluses is greater than or equal to the number of minuses minus two. To see that this condition is satisfied in $M_{\mathcal{J}}$ note that since $\mathcal{I}^{+}=\{i\}$ we have that the total number of positive helicity gravitons in $M_{\mathcal{J}}$ is $p-1$ while that of negative helicity gravitons is $m-m_{\mathcal{I}}+1$. Since the number of external negative helicity gravitons in $M_{\mathcal{I}}$ must be at least one, i.e, $m_{\mathcal{I}} \geq 1$ and recalling that we are studying the case when $p \geq m$, we get the desired result.

The next case to consider is when $h=-$. Since $P_{\mathcal{I}}^{+}\left(w_{\mathcal{I}}\right)$ is $z$ independent, the deformation (3.43) of $M_{\mathcal{J}}$ is no longer maximal. However, it is possible to show that these terms are identically zero. This is obvious when the on-shell physical amplitude $M_{\mathcal{I}}$, which has only one positive helicity graviton, has more than two negative helicity gravitons.

Consider now the case when $M_{\mathcal{I}}$ has precisely two negative helicity gravitons. A three-graviton on-shell amplitude need not vanish if momenta are complex therefore this is a potentially dangerous case. Three-graviton amplitudes are given as the square of the gauge theory ones. Therefore we have

$$
M_{\mathcal{I}}\left(i^{+}\left(w_{\mathcal{I}}\right), s^{-},-P_{\mathcal{I}}^{-}\left(w_{\mathcal{I}}\right)\right)=\left(\frac{\left\langle\lambda^{(s)}, \lambda^{(P)}\right\rangle^{3}}{\left\langle\lambda^{(P)}, \lambda^{(i)}\left(w_{\mathcal{I}}\right)\right\rangle\left\langle\lambda^{(i)}\left(w_{\mathcal{I}}\right), \lambda^{(s)}\right\rangle}\right)^{2}
$$

where as in section III.C we have defined $P_{\mathcal{I}}\left(w_{\mathcal{I}}\right)_{a \dot{a}}=\lambda_{a}^{(P)} \tilde{\lambda}_{\dot{a}}^{(P)}$.

Since this is a physical amplitude, momentum is conserved which means

$$
\lambda^{(i)}\left(w_{\mathcal{I}}\right)_{a} \tilde{\lambda}_{\dot{a}}^{(i)}+\lambda_{a}^{(s)} \tilde{\lambda}_{\dot{a}}^{(s)}=\lambda_{a}^{(P)} \tilde{\lambda}_{\dot{a}}^{(P)} .
$$

For real momenta, this equation implies that all $\lambda^{\prime} s$ and all $\tilde{\lambda}^{\prime} s$ are proportional. Therefore three-graviton amplitudes must vanish. For complex momenta, this need not be the case and one can have all $\tilde{\lambda}^{\prime} s$ be proportional with the $\lambda$ 's unconstrained. In such a case (3.44) would not vanish.

We claim that, luckily in our case of interest, all $\lambda^{\prime} s$ are proportional and (3.44) vanishes. To see this note that $w_{\mathcal{I}}=-\langle i, s\rangle /\langle j, s\rangle$ and $\lambda^{(i)}\left(w_{\mathcal{I}}\right)_{a}=\lambda_{a}^{(i)}+w_{\mathcal{I}} \lambda_{a}^{(j)}$, therefore 
$\left\langle\lambda^{(i)}\left(w_{\mathcal{I}}\right), \lambda^{(s)}\right\rangle=0$. Contracting (3.45) with $\lambda^{(s) a}$ we find $\left\langle\lambda^{(P)}, \lambda^{(s)}\right\rangle \tilde{\lambda}_{\dot{a}}^{(P)}=0$. Therefore we must have $\left\langle\lambda^{(P)}, \lambda^{(s)}\right\rangle=0$ which completes the proof of our claim.

From (3.44), this condition implies that $M_{\mathcal{I}}$ is identically zero. Thus, we can conclude that the cases of $M_{\mathcal{J}}$ with a non-maximal deformation are not there.

This is the end of our proof. We now turn to some extensions and applications of the $\mathrm{BCFW}$ recursion relations that can be obtained by using Ward identities.

\section{Ward Identities}

Our proof of the BCFW recursion relations was based on deforming two gravitons of opposite helicities, $i^{+}$and $j^{-}$, in the following way:

$$
\lambda^{(i)}(z)=\lambda^{(i)}+z \lambda^{(j)}, \quad \tilde{\lambda}^{(j)}(z)=\tilde{\lambda}^{(j)}-z \tilde{\lambda}^{(i)} .
$$

However, it is known that in gauge theory, deformed amplitudes also vanish at infinity if the helicities $\left(h_{i}, h_{j}\right)$ of the deformed gluons are $(-,-)$ or $(+,+)[11]$. It would be interesting to prove a similar statement for General Relativity. Here we show that this is indeed very straightforward in the case of MHV scattering amplitudes if one uses Ward identities.

The Ward identity of relevance for our discussion can be found for example in [41] and it is given by

$$
\frac{M_{l, m}^{\mathrm{MHV}}}{\left\langle\lambda^{(l)}, \lambda^{(m)}\right\rangle^{8}}=\frac{M_{s, q}^{\mathrm{MHV}}}{\left\langle\lambda^{(s)}, \lambda^{(q)}\right\rangle^{8}},
$$

where the notation $M_{a, b}^{\mathrm{MHV}}$ indicates that the gravitons $a$ and $b$ in this amplitude are the ones with negative helicity.

Consider first the $(+,+)$ case. We use the Ward identity (4.2) to relate it to the usual $(+,-)$ case. For clarity purposes, we explicitly exhibit the dependence of the amplitudes on only four gravitons: $\{l, m, i, j\}$. The dependence on the rest of the gravitons (all of which have positive helicity) will be implicit. Then we have

$$
M_{n}^{\mathrm{MHV}}\left(i^{+}(z), j^{+}(z), l^{-}, m^{-}\right)=\left(\frac{\left\langle\lambda^{(l)}, \lambda^{(m)}\right\rangle}{\left\langle\lambda^{(j)}, \lambda^{(l)}\right\rangle}\right)^{8} M_{n}^{\mathrm{MHV}}\left(i^{+}(z), j^{-}(z), l^{-}, m^{+}\right) .
$$

The MHV amplitude on the right hand-side is deformed as in (4.1), thus it vanishes at infinity by our proof. Since both inner products expressed explicitly in (4.3) do not depend on $z$, the amplitude on the left hand side of $(4.3)$, where $\left(h_{i}, h_{j}\right)=(+,+)$, will vanish as $z$ goes to infinity.

Consider now the $(-,-)$ case. Using again the Ward identity (4.2) we have

$$
M_{n}^{\mathrm{MHV}}\left(i^{-}(z), j^{-}(z), l^{+}, m^{+}\right)=\left(\frac{\left\langle\lambda^{(i)}(z), \lambda^{(j)}\right\rangle}{\left\langle\lambda^{(j)}, \lambda^{(l)}\right\rangle}\right)^{8} M_{n}^{\mathrm{MHV}}\left(i^{+}(z), j^{-}(z), l^{-}, m^{+}\right)
$$

Note that $\left\langle\lambda^{(i)}(z), \lambda^{(j)}\right\rangle$ does not depend on $z$ since $\lambda^{(i)}(z)=\lambda^{(i)}+z \lambda^{(j)}$. Therefore, the amplitude still vanishes in this case.

In [21], a very nice compact formula was conjectured for MHV amplitudes of gravitons by assuming the validity of $\mathrm{BCFW}$ recursion relations obtained via a deformation of the 
two negative helicity gravitons. Our proof and the discussion in this section validates the recursion relations used to construct the all multiplicity ansatz. It would be highly desirable to show that the formula proposed by Bedford et al. [21] does indeed satisfy the recursion relations. The formula is explicitly given by

$M_{n}\left(1^{-}, 2^{-}, i_{1}^{+}, \ldots, i_{n-2}^{+}\right)=\frac{\langle 1,2\rangle^{6}\left[1, i_{n-2}\right]}{\left\langle 1, i_{n-2}\right\rangle} G\left(i_{1}, i_{2}, i_{3}\right) \prod_{s=3}^{n-3} \frac{\left\langle 2\left|i_{1}+\ldots+i_{s-1}\right| i_{s}\right]}{\left\langle i_{s}, i_{s+1}\right\rangle\left\langle 2, i_{s+1}\right\rangle}+\mathcal{P}\left(i_{1}, \ldots, i_{n-2}\right)$

where $\mathcal{P}\left(i_{1}, \ldots, i_{n-2}\right)$ indicates a sum over all permutations of $\left(i_{1}, \ldots, i_{n-2}\right)$ and

$$
G\left(i_{1}, i_{2}, i_{3}\right)=\frac{1}{2}\left(\frac{\left[i_{1}, i_{2}\right]}{\left\langle 2, i_{1}\right\rangle\left\langle 2, i_{2}\right\rangle\left\langle i_{1}, i_{2}\right\rangle\left\langle i_{2}, i_{3}\right\rangle\left\langle i_{1}, i_{3}\right\rangle}\right) .
$$

It is also interesting to show why the case $\left(h_{i}, h_{j}\right)=(-,+)$ does not lead to recursion relations. Using the Ward identity (4.2) once again we have

$$
M_{n}^{\mathrm{MHV}}\left(i^{-}(z), j^{+}(z), l^{+}, m^{-}\right)=\left(\frac{\left\langle\lambda^{(i)}(z), \lambda^{(m)}\right\rangle}{\left\langle\lambda^{(i)}(z), \lambda^{(j)}\right\rangle}\right)^{8} M_{n}^{\mathrm{MHV}}\left(i^{-}(z), j^{-}(z), l^{+}, m^{+}\right)
$$

The amplitude on the right hand-side vanishes as $z$ goes to infinity. However, $\left\langle\lambda^{(i)}(z), \lambda^{(m)}\right\rangle^{8}$ contributes with a factor of $z^{8}$ while $\left\langle\lambda^{(i)}(z), \lambda^{(j)}\right\rangle$ is $z$ independent. Either using BGK (together with (4.4)) or directly (4.5), one can show that $M_{n}^{\mathrm{MHV}}\left(i^{-}(z), j^{-}(z), l^{+}, m^{+}\right)$goes like $1 / z^{2}$, therefore the amplitude with $\left(h_{i}, h_{j}\right)=(-,+)$ behaves as $z^{6}$ at infinity.

\section{Conclusions And Further Directions}

In this paper we have proven that tree level gravity amplitudes in General Relativity are very special. Contrary to what can be called a naive power counting of the behavior of individual Feynman diagrams, full amplitudes actually vanish when momenta are taken to infinity along some complex direction. The naive power counting gives that the amplitudes diverge. This miraculous property implies that tree amplitudes of gravitons satisfy a special kind of recursion relations. One in which an amplitude is given as a sum of terms containing the product of two physical on-shell amplitudes where the momenta of only two gravitons have been complexified. These recursion relations, originally discovered in [7] in gauge theory, were proven using the power of complex analysis in [11]. The BCFW construction opened up the possibility for using complex analysis in many other situations. There are only two major difficulties when applying the BCFW construction to a general field theory at any order in perturbation theory. One of them is that complete control of the singularity structure of the amplitude is required. At tree-level this means poles but at the loop level one can also have branch cuts. The other one is to have a good control on the behavior at infinity. In the case of gravity amplitudes this had been the stumbling block. The way we overcome this obstacle was by constructing auxiliary recursion relations. These were obtained by exploiting as many polarization tensors as possible in other to tame the divergent behavior of vertices in individual Feynman diagrams while still keeping the 
linear behavior of propagators. In a sense, the deformation we introduced is the "maximal" choice.

This procedure seems quite general and it would be very interesting to classify field theories according to whether their amplitudes vanish or not at infinity under this maximal deformation.

\section{Note Added (November 23, 2008):}

In the original version of this paper we made a wrong statement in the introduction. We claimed that the deformation of [34] follows from the basic BCFW deformation proven in this paper. However, the precise statement made in [34] is that a proof of the basic BCFW deformation would provide evidence for their deformation. In fact, it has recently been proven in [36] by using direct numerical analysis that the deformation of [34] generically fails to vanish at infinity for $n \geq 12$ which means that the validity of the basic BCFW deformation is a necessary but not sufficient condition for the validity of the non-maximal deformations. The precise result found in [36] is for NMHV amplitudes and states that under a Risager deformation of the three negative helicity gravitons the large $z$ behavior is given by $z^{n-12}$. Given that this result might have important consequences we applied some of the techniques in this paper to construct an analytic proof. We provide the analytic proof of such behavior in appendix B.

\section{Acknowledgments}

PB would like to thank Perimeter Institute for hospitality during a visit where part of this research was done. PB is also grateful to Fiorenzo Bastianelli for hospitality at the Department of Physics, University of Bologna. The research of CBV and FC at Perimeter Institute for Theoretical Physics is supported in part by the Government of Canada through NSERC and by the Province of Ontario through MRI. CBV also acknowledges support from NSERC Canadian Graduate Scholarship.

\section{A. Proof Of Auxiliary Recursion Relations}

In the main part of the paper we used certain auxiliary recursion relations to prove that $M_{n}(z)$ vanishes as $z$ is taken to infinity under the BCFW deformation. It is therefore very important to establish the validity of the auxiliary recursion relations.

Consider the case when then number of positive helicity gravitons is larger or equal than the number of negative helicity ones, i.e, $p \geq m$. The case when $m \geq p$ is completely analogous. Let us start by constructing a rational function $M_{n}(w)$ of a complex variable $w$ via the deformation (3.7), i.e,

$$
\tilde{\lambda}^{(j)}(w)=\tilde{\lambda}^{(j)}-w \sum_{s \in\left\{k^{+}\right\}} \tilde{\lambda}^{(s)}, \quad \lambda^{(k)}(w)=\lambda^{(k)}+w \lambda^{(j)}, \quad \forall k \in\left\{k^{+}\right\}
$$

where $j$ is a negative helicity graviton and $\left\{k^{+}\right\}$is the set of all positive helicity gravitons in $M_{n}$.

The claim is that $M_{n}(w)$ vanishes as $w$ is taken to infinity and its only singularities are simple poles at finite values of $w$. 


\section{A.1 Vanishing Of $M_{n}(w)$ At Infinity}

Let us prove that $M_{n}(w)$ vanishes as $w \rightarrow \infty$. Consider the leading Feynman diagram that contributes to $M_{n}(w)$. Such a diagram has $n-2$ cubic vertices each contributing a factor of $w^{2}$. It also has $p+1$ polarization tensors that depend on $w$ and give $1 / w^{2}$ each. Finally, we claim that all $n-3$ propagators that can possibly depend on $w$ actually do giving each a contribution of $1 / w$. Putting all contributions together we find that the leading Feynman diagrams go like $1 / w^{p-m+3}$. Therefore, if $p \geq m$ then $M_{n}(w) \rightarrow 0$ as $w \rightarrow \infty$.

We are only left to prove that $n-3$ propagators depend on $w$. A similar statement has to be proven in section III.D. The proof there is more involved since it requires the study of many cases. The discussion that follows can be thought of as a warm up for that in section III.D.

Consider a given Feynman diagram. A propagator naturally divides the diagram into two sub-diagrams. Let us denote them by $\mathcal{I}$ and $\mathcal{J}$. Without loss of generality, we can always take the $j^{\text {th }}$ graviton to be in $\mathcal{J}$. Let us denote the set of positive helicity gravitons in $\mathcal{I}$ by $\mathcal{I}^{+}$.

The propagator under consideration has the form $1 / P_{\mathcal{I}}^{2}(w)$ with

$$
P_{\mathcal{I}}^{2}(w)=P_{\mathcal{I}}^{2}-w \sum_{k \in \mathcal{I}^{+}}\left\langle j\left|P_{\mathcal{I}}\right| k\right]
$$

where $P_{\mathcal{I}}=P_{\mathcal{I}}(0)$.

The only way the $w$ dependence can drop out of the propagator is that $\sum_{k \in \mathcal{I}^{+}}\left\langle j\left|P_{\mathcal{I}}\right| k\right]=$ 0 .

Since the $j^{\text {th }}$ graviton belongs to $\mathcal{J}$, the condition $\sum_{k \in \mathcal{I}^{+}}\left\langle j\left|P_{\mathcal{I}}\right| k\right]=0$ can only be satisfied if the vector $\sum_{k \in \mathcal{I}^{+}} P_{\mathcal{I} a \dot{a}} \tilde{\lambda}^{(k) \dot{a}}$ vanishes. To see this note that there must be at least two gravitons in $\mathcal{J}$, one of them $j$. Therefore we can use momentum conservation to determine the other one in terms of the other $n-1$ gravitons. This allows us to consider all the remaining $n-1$ gravitons as independent. In particular, the $j^{\text {th }}$ graviton is independent from the ones in $\mathcal{I}$.

Our goal is then to prove that the combination $P_{\mathcal{I} a \dot{a}}\left(\sum_{k \in \mathcal{I}^{+}} \tilde{\lambda}_{\dot{a}}^{(k)}\right)$ cannot vanish for generic choice of momenta and polarization tensors.

Consider first the case when the set $\mathcal{I}^{+}$has only one element, say the $s^{\text {th }}$ graviton. Then the vanishing of $P_{\mathcal{I} a \dot{a}} \tilde{\lambda}^{(s) \dot{a}}$ implies that of $\sum_{k \in \mathcal{I}} s_{k, s}$, where $s_{k, s}=\left(p_{k}+p_{s}\right)^{2}$. Since $\mathcal{I}$ must have at least two gravitons, the vanishing of $\sum_{k \in \mathcal{I}} s_{k, s}$ is a constraint on the kinematical invariants which is not satisfied for generic momenta.

Consider the case when $\mathcal{I}^{+}$has at least two elements. Let one of them be the $s^{\text {th }}$ graviton. Since our starting point is a physical on-shell amplitude, the dependence of the amplitude on the $s^{\text {th }}$ graviton can only be through its polarization tensor and its momentum vector,

$$
\epsilon_{a \dot{a}, b \dot{b}}^{+(s)}=\frac{\mu_{a} \tilde{\lambda}_{\dot{a}}^{(s)} \mu_{b} \tilde{\lambda}_{\dot{b}}^{(s)}}{\left\langle\mu, \lambda^{(s)}\right\rangle^{2}}, \quad p_{a \dot{a}}^{(s)}=\lambda_{a}^{(s)} \tilde{\lambda}_{\dot{a}}^{(s)} .
$$

If we transform $\left\{\lambda^{(s)}, \tilde{\lambda}^{(s)}\right\}$ into $\left\{t^{-1} \lambda^{(s)}, t \tilde{\lambda}^{(s)}\right\}$ with $t^{4}=1$, i.e., $t$ is any $4^{\text {th }}$ root of unity, then both $\epsilon_{a \dot{a}, b \dot{b}}^{+(s)}$ and $p_{a \dot{a}}^{(s)}$ are invariant. This means that any statement we make for $t=1$ 
must be true for the other three possible values of $t$. In particular, it must be the case that $P_{\mathcal{I} a \dot{a}}\left(\sum_{k \in \mathcal{I}^{+}, k \neq s} \tilde{\lambda}_{\dot{a}}^{(k)}+t \tilde{\lambda}_{\dot{a}}^{(s)}\right)$ vanishes for all four values of $t$. Since $P_{\mathcal{I} a \dot{a}}$ does not depend on $t$ the only way to satisfy this condition is if $P_{\mathcal{I}} \cdot p^{(s)}=0$. This is clearly a condition that is not satisfied for generic momenta and therefore this possibility is also excluded.

Finally, there is one more possibility to consider. If the set $\mathcal{I}^{+}$is empty then the $w$ dependence drops out. Of course, this is not a problem because if the set $\mathcal{I}^{+}$is empty it means that nothing on the subdiagram $\mathcal{I}$ depends on $w$, including the cubic vertices. Therefore, neither propagators nor cubic vertices contribute. One can then concentrate on the subdiagram $\mathcal{J}$, but this subdiagram has less particles than the total diagram and the same number of $w$-dependent polarization tensor. Therefore these diagrams go to zero even faster than diagrams where $\mathcal{I}^{+}$is not empty.

\section{A.2 Location Of Poles And Final Form Of The Auxiliary Recursion Relations}

Having proven that $M_{n}(w)$ vanishes at infinity, we turn to the question of the singularity structure. We claim that it has only simple poles coming from propagators in Feynman diagrams. Again as in section II where we discussed the BCFW deformation, one has that the poles generated by the $w$ dependence in the polarization tensors can be eliminated by a gauge choice. We pick the reference spinor of each of the polarization tensors of the positive helicity gravitons to be $\mu_{a}=\lambda_{a}^{(j)}$ and that of the $j^{\text {th }}$ helicity graviton to be $\tilde{\mu}_{\dot{a}}=\sum_{k \in\left\{k^{+}\right\}} \tilde{\lambda}^{(k)}$.

We have already given the structure of propagators in (A.2) from where we can immediately read off the location of the poles to be

$$
w_{\mathcal{I}}=\frac{P_{\mathcal{I}}^{2}(0)}{\sum_{k \in \mathcal{I}^{+}}\left\langle j\left|P_{\mathcal{I}}(0)\right| k\right]} .
$$

Finally, we need the fact that a rational function that vanishes at infinity and only has simple poles can be written as $M_{n}(w)=\sum_{\alpha} c_{\alpha} /\left(w-w_{\alpha}\right)$ where the sum is over the poles and $c_{\alpha}$ are the residues. The residues in this case can be determined from factorization limits since all poles come from physical propagators.

Collecting all results we arrive at the final form of the auxiliary recursion relation used in the text (3.9):

$$
\begin{aligned}
& M_{n}\left(\left\{r^{-}\right\},\left\{k^{+}\right\}\right)= \\
& \quad=\sum_{\mathcal{I}} \sum_{h= \pm} M_{\mathcal{I}}\left(\left\{r_{\mathcal{I}}^{-}\right\},\left\{k_{\mathcal{I}}^{+}\left(w_{\mathcal{I}}\right)\right\},-P_{\mathcal{I}}^{h}\left(w_{\mathcal{I}}\right)\right) \frac{1}{P_{\mathcal{I}}^{2}} M_{\mathcal{J}}\left(\left\{r_{\mathcal{J}}^{-}\left(w_{\mathcal{I}}\right)\right\},\left\{k_{\mathcal{J}}^{+}\left(w_{\mathcal{I}}\right)\right\}, P_{\mathcal{I}}^{-h}\left(w_{\mathcal{I}}\right)\right) .
\end{aligned}
$$

\section{B. Large- $z$ behavior of NMHV gravity amplitudes under a three-particle deformation}

In [34] the MHV-expansion for gravity scattering amplitude was discussed. The main idea was to obtain it as a recursion relation: it was argued that the suitable one-parameter 
deformation of the momentum space which could allow to explicitly obtain such an expansion can be introduced by shifting three gravitons with same helicity as follows. For semplicity, let us consider an NMHV amplitude and let us deform the momenta of three negative helicity gravitons $\{1,2,3\}$

$$
\tilde{\lambda}^{(i)}(z)=\tilde{\lambda}^{(i)}-z\langle j, k\rangle \tilde{\eta}, \quad i, j, k=1,2,3,
$$

where $\tilde{\eta}$ is a reference spinor and the coefficients $\langle j, k\rangle$ are fixed by momentum conservation (the indices $i, j, k$ assume the values $1,2,3$ cyclically). It was assumed that the deformed amplitude was well-behaved as $z \rightarrow \infty$, which is the necessary and sufficient condition to determine the amplitude only from its poles at finite points. Recently, it was shown in [36] that actually this condition does not hold for an arbitrary number $n$ of external gravitons. More precisely, it has been shown numerically that, under the deformation (B.1), the NMHV graviton amplitude vanishes as long as the number of external gravitons is less than 12:

$$
M_{n}^{\mathrm{NMHV}}(z) \sim \frac{1}{z^{n-12}}
$$

In this section we provide an analitycal proof of such behavior. The main idea of the following proof is to consider a suitable representation for the NMHV graviton amplitude and then apply the three-particle deformation (B.1). Such a representation is obtained through a BCFW-like deformation of two positive helicity gravitons, which we label by 4 and 5. It is important to mention that the validity of such a deformation does not follow from the one proven in this paper where opposite helicity gravitons are deformed. Luckily, a proof that equal helicity gravitons can also be defomed was recently given by ArkaniHamed and Kaplan in [42]. As shown in Figure 1, this particular deformation induces a diagrammatic expansion for the amplitude with 6 types of contributions.

$$
\begin{aligned}
& M_{n}=\sum_{l \in \hat{\mathcal{I}}^{+}} M_{n-1}\left(\left\{r^{-}\right\},\left\{\hat{\mathcal{I}}_{l}^{+}\right\}, \hat{4}^{+}, \hat{P}_{l 5}^{+}\right) \frac{1}{P_{l 5}^{2}} M_{3}\left(-\hat{P}_{l 5}^{-}, l^{+}, \hat{5}^{+}\right)+ \\
& +\sum_{i, j, k \in\{1,2,3\}} M_{n-1}\left(i^{-}, j^{-},\left\{\hat{\mathcal{I}}^{+}\right\}, \hat{4}^{+}, \hat{P}_{k 5}^{-}\right) \frac{1}{P_{k 5}^{2}} M_{3}\left(-\hat{P}_{k 5}^{+}, k^{-}, \hat{5}^{+}\right)+ \\
& +\sum_{\hat{\mathcal{I}}^{+}} \sum_{i, j, k \in\{1,2,3\}} M_{\hat{\mathcal{J}}^{+}}\left(i^{-}, j^{-},\left\{\hat{\mathcal{J}}^{+}\right\}, \hat{4}^{+}, \hat{P}_{\hat{\mathcal{I}}^{+}}\right) \frac{1}{P_{\hat{\mathcal{I}}^{+}}^{2}} M_{\hat{\mathcal{I}}^{+}}\left(-\hat{P}_{\hat{\mathcal{I}}}^{-},\left\{\hat{\mathcal{I}}^{+}\right\}, k^{-}, \hat{5}^{+}\right)+ \\
& +\sum_{\hat{\mathcal{I}}^{+}} \sum_{i, j, k \in\{1,2,3\}} M_{\hat{\mathcal{I}}^{+}}\left(i^{-},\left\{\hat{\mathcal{I}}^{+}\right\}, \hat{4}^{+},-\hat{P}_{\hat{\mathcal{I}}^{-}}\right) \frac{1}{P_{\hat{\mathcal{I}}^{+}}^{2}} M_{\hat{\mathcal{J}}^{+}}\left(\hat{P}_{\hat{\mathcal{I}}^{+}},\left\{\hat{\mathcal{J}}^{+}\right\}, j^{-}, k^{-}, \hat{5}^{+}\right)+ \\
& +\sum_{i, j, k \in\{1,2,3\}} M_{3}\left(i^{-}, \hat{4}^{+},-\hat{P}_{i 4}^{+}\right) \frac{1}{P_{i 4}^{2}} M_{n-1}\left(\hat{P}_{i 4}^{-}, j^{-}, k^{-},\left\{\hat{\mathcal{J}}^{+}\right\} \hat{5}^{+}\right)+ \\
& +\sum_{l \in \hat{\mathcal{I}}^{+}} M_{3}\left(l^{+}, \hat{4}^{+},-\hat{P}_{l 4}^{-}\right) \frac{1}{P_{l 4}^{2}} M_{n-1}\left(\left\{r^{-}\right\},\left\{\hat{\mathcal{I}}_{l}^{+}\right\}, \hat{5}^{+}, \hat{P}_{l 4}^{+}\right),
\end{aligned}
$$

where $\hat{\mathcal{I}}^{+} \equiv \mathcal{I}^{+} \backslash\left\{4^{+}, 5^{+}\right\}$and $\hat{\mathcal{I}}_{l}^{+} \equiv \hat{\mathcal{I}}^{+} \backslash\left\{l^{+}\right\}$. Let us write explicitly down the deformation 


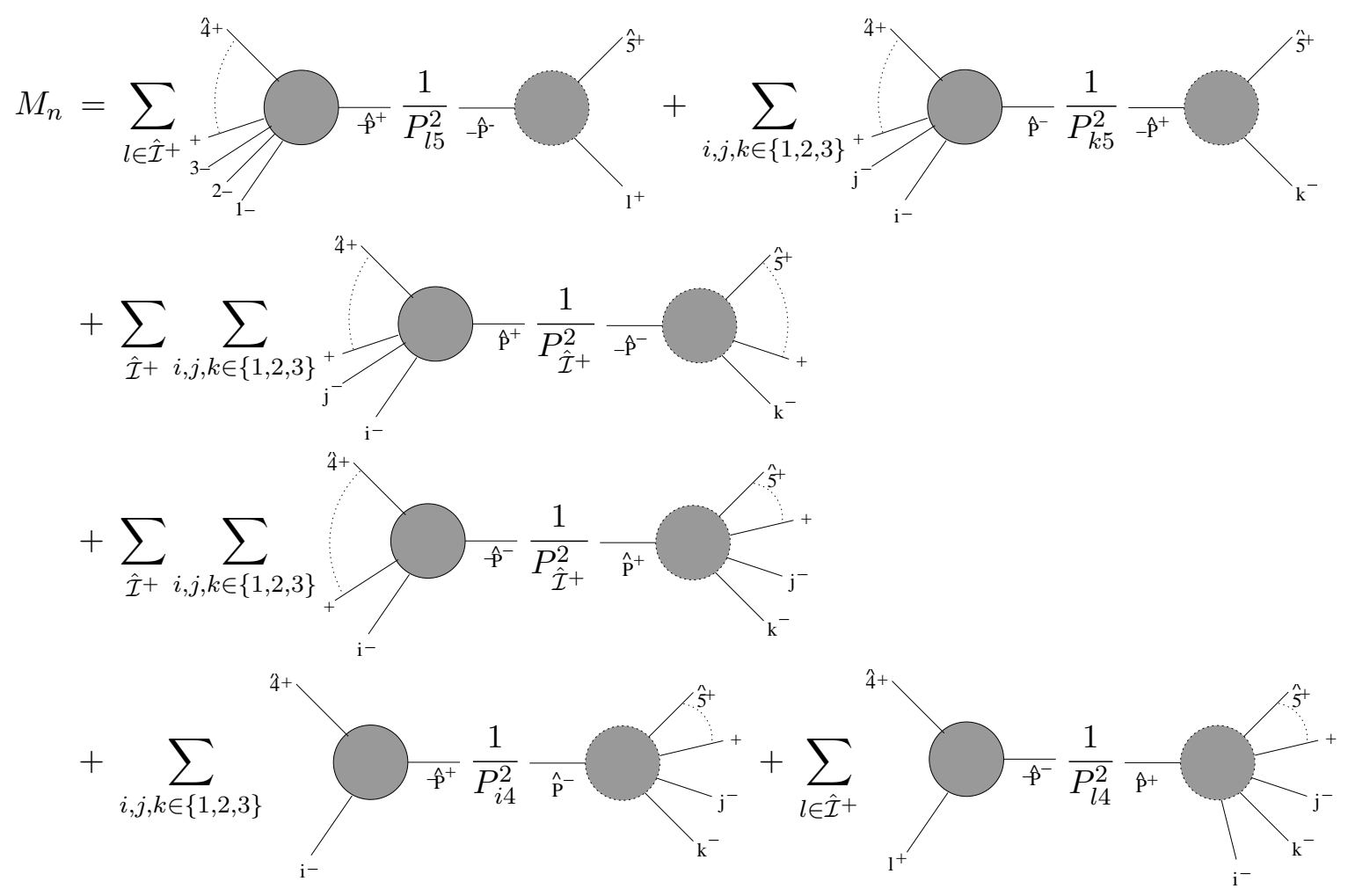

Figure 1: Representation of the NMHV gravity amplitude under a BCFW-like deformation of momenta of two positive helicity gravitons

we are using

$$
\tilde{\lambda}^{(5)}(w)=\tilde{\lambda}^{(5)}-w \tilde{\lambda}^{(4)}, \quad \lambda^{(4)}(w)=\lambda^{(4)}+w \lambda^{(5)} .
$$

It is possible to see immediately that the first two lines in (B.3) vanish, given that the threeparticle amplitudes present are zero: those three-particle amplitudes are anti-holomorphic and the anti-holomorphic spinors involved are proportional to each other, so that $M_{3}=0$.

Let us now consider the terms in the last line of (B.3). The poles in this channel are located at $w_{l 4}=\langle l, 4\rangle /\langle 5, l\rangle$, and the deformed spinors evaluated at these poles are given by

$$
\hat{\tilde{\lambda}}^{(5)}=\tilde{\lambda}^{(5)}-\frac{\langle l, 4\rangle}{\langle 5, l\rangle} \tilde{\lambda}^{(4)}, \quad \hat{\lambda}^{(4)}=\frac{\langle 5,4\rangle}{\langle 5, l\rangle} \lambda^{(l)} .
$$

Similarly, the spinors related to on-shell "internal" momentum $P_{l 4}$ can be written as

$$
\lambda^{(P)}=\lambda^{(l)}, \quad \tilde{\lambda}^{(P)}=\tilde{\lambda}^{(l)}+\frac{\langle 5,4\rangle}{\langle 5, l\rangle} \tilde{\lambda}^{(4)} .
$$

It is straightforward then to see that, under the deformation (B.1) both the propagator $1 / P_{l 4}^{2}$ and the three-particle amplitude are $z$-independent, while the $(n-1)$-particle amplitude gets deformed in the same way of the original amplitude. These terms can therefore be treated by induction. 
We repeat the same strategy for all the other terms in the BCFW-representation, starting with the fifth line in (B.3). In this channel the poles are located at $w_{i 4}=\langle i, 4\rangle /\langle 5, i\rangle$, and the deformed spinors evaluated at these poles writes

$$
\hat{\tilde{\lambda}}^{(5)}=\tilde{\lambda}^{(5)}-\frac{\langle i, 4\rangle}{\langle 5, i\rangle} \tilde{\lambda}^{(4)}, \quad \hat{\lambda}^{(4)}=\frac{\langle 5,4\rangle}{\langle 5, i\rangle} \lambda^{(i)},
$$

with

$$
\lambda^{(P)}=\lambda^{(i)}, \quad \tilde{\lambda}^{(P)}=\tilde{\lambda}^{(i)}+\frac{\langle 5,4\rangle}{\langle 5, i\rangle} \tilde{\lambda}^{(4)} .
$$

Contrarly to the previous case, here the three-particle deformation (B.1) induces a nontrivial $z$-dependence in (B.8) given that there is an explicit dependence on one of the deformed spinors (namely the one labeled by $i$ ). It is interesting to notice the way they get deformed:

$$
\lambda^{(P)}(z)=\lambda^{(P)}, \quad \tilde{\lambda}^{(P)}(z)=\tilde{\lambda}^{(P)}-z\langle j, k\rangle \tilde{\eta},
$$

which implies that for the three-particle amplitude we have:

$$
M_{3}\left(i^{-}, \hat{4}^{+},-\hat{P}_{i 4}^{+}\right)=\left(\frac{[4, P(z)]^{3}}{[P(z), i(z)][i(z), 4]}\right)^{2}=\left(\frac{\langle 5, i\rangle}{\langle 5,4\rangle}([4, i]-z\langle j, k\rangle[4, \tilde{\eta}])\right)^{2} \sim z^{2}
$$

while the propagator $1 / P_{i 4}^{2}(z)$ behaves as $1 / z$ and $(n-1)$-particle amplitude again is deformed according to a shift of type (B.1). This means that the product of the threeparticle amplitude and the propagator behaves as $\sim z$ for large $z$.

Note that this is very different from the behavior in Yang-Mills where the three-particle amplitude is the square root of that in gravity and its large $z$ behavior is canceled by that of the propagator. This means that in Yang-Mills the same product goes like 1 for large $z$.

In order to conclude the argument, we simply have to notice that the amplitude multiplying the $\mathcal{O}(z)$ term in gravity (and $\mathcal{O}(1)$ term in Yang-Mills) is a $(n-1)$-particle amplitude under the three-particle deformation (B.1). This means that if the $(n-1)$ particle amplitude behaves as $z^{a_{n-1}}$ at infinity then the $n$-particle amplitude behaves as $z^{a_{n-1}+1}$ in gravity (and as $z^{a_{n-1}}$ in Yang-Mills). All we need is to find out what $a_{n}$ is and this can be done by studying explicitly low point amplitudes. Using the explicit form of the amplitude, we have checked that a five particle amplitude behaves as $z^{-7}$. Therefore $a_{5}=-7$. Using that $a_{n}=a_{n-1}+1$ we find $a_{n}=n-12$ which is what we wanted to prove. In Yang-Mills one finds that $a_{n}=a_{n-1}$ and therefore the amplitude vanishes at infinity if it does for any $n$. It is easy to check that this is indeed the case for $n=5$.

\section{References}

[1] F. A. Berends and W. T. Giele, "Recursive calculations for processes with $n$ gluons," Nucl. Phys. B 306, 759 (1988).

[2] M. L. Mangano, S. J. Parke and Z. Xu, "Duality and multi-gluon scattering," Nucl. Phys. B 298, 653 (1988). 
[3] M. L. Mangano and S. J. Parke, "Multiparton amplitudes in gauge theories," Phys. Rept. 200, 301 (1991) hep-th/0509223.

[4] F. A. Berends, W. T. Giele and H. Kuijf, "Exact and approximate expressions for multi-gluon scattering," Nucl. Phys. B 333, 120 (1990).

[5] D. A. Kosower, "Light cone recurrence relations for QCD amplitudes," Nucl. Phys. B 335, 23 (1990).

[6] S. J. Parke and T. R. Taylor, "An Amplitude For N Gluon Scattering," Phys. Rev. Lett. 56, 2459 (1986).

[7] R. Britto, F. Cachazo and B. Feng, "New recursion relations for tree amplitudes of gluons," Nucl. Phys. B 715 (2005) 499 hep-th/0412308.

[8] R. Britto, F. Cachazo and B. Feng, "Generalized unitarity and one-loop amplitudes in N =4 super-Yang-Mills," Nucl. Phys. B 725, 275 (2005) hep-th/0412103.

[9] R. Roiban, M. Spradlin and A. Volovich, "Dissolving $\mathrm{N}=4$ loop amplitudes into QCD tree amplitudes," Phys. Rev. Lett. 94, 102002 (2005) hep-th/0412265.

[10] Z. Bern, V. Del Duca, L. J. Dixon and D. A. Kosower, "All non-maximally-helicity-violating one-loop seven-gluon amplitudes in $\mathrm{N}=4$ super-Yang-Mills theory," Phys. Rev. D 71, 045006 (2005) hep-th/0410224.

[11] R. Britto, F. Cachazo, B. Feng and E. Witten, "Direct proof of tree-level recursion relation in Yang-Mills theory," Phys. Rev. Lett. 94 (2005) 181602 hep-th/0501052.

[12] M. x. Luo and C. k. Wen, "Recursion relations for tree amplitudes in super gauge theories," JHEP 0503, 004 (2005) hep-th/0501121.

[13] M. x. Luo and C. k. Wen, "Compact formulas for all tree amplitudes of six partons," Phys. Rev. D 71, 091501 (2005) hep-th/0502009.

[14] Z. Bern, L. J. Dixon and D. A. Kosower, "The last of the finite loop amplitudes in QCD," Phys. Rev. D 72, 125003 (2005) hep-ph/0505055.

[15] Z. Bern, L. J. Dixon and D. A. Kosower, "Bootstrapping multi-parton loop amplitudes in QCD," Phys. Rev. D 73, 065013 (2006) hep-ph/0507005.

[16] C. F. Berger, Z. Bern, L. J. Dixon, D. Forde and D. A. Kosower, "Bootstrapping one-loop QCD amplitudes with general helicities," Phys. Rev. D 74, 036009 (2006) hep-ph/0604195.

[17] C. F. Berger, Z. Bern, L. J. Dixon, D. Forde and D. A. Kosower, "All one-loop maximally helicity violating gluonic amplitudes in QCD," hep-ph/0607014.

[18] Z. Bern, N. E. J. Bjerrum-Bohr, D. C. Dunbar and H. Ita, "Recursive calculation of one-loop QCD integral coefficients," JHEP 0511, 027 (2005) hep-ph/0507019.

[19] A. Brandhuber, S. McNamara, B. Spence and G. Travaglini, "Recursion relations for one-loop gravity amplitudes," hep-th/0701187.

[20] S. D. Badger, E. W. N. Glover, V. V. Khoze and P. Svrcek, "Recursion relations for gauge theory amplitudes with massive particles," JHEP 0507, 025 (2005) hep-th/0504159.

[21] J. Bedford, A. Brandhuber, B. J. Spence and G. Travaglini, "A recursion relation for gravity amplitudes," Nucl. Phys. B 721, 98 (2005) hep-th/0502146. 
[22] F. Cachazo and P. Svrcek, "Tree level recursion relations in general relativity," hep-th/0502160.

[23] F. A. Berends, W. T. Giele and H. Kuijf, "On relations between multi - gluon and multigraviton scattering," Phys. Lett. B 211 (1988) 91.

[24] H. Kawai, D. C. Lewellen and S. H. H. Tye, "A Relation Between Tree Amplitudes Of Closed And Open Strings," Nucl. Phys. B 269 (1986) 1.

[25] M. B. Green, J. G. Russo and P. Vanhove, "Non-renormalisation conditions in type II string theory and maximal supergravity," hep-th/0610299.

[26] Z. Bern, L. J. Dixon and R. Roiban, "Is N = 8 supergravity ultraviolet finite?," Phys. Lett. B 644, 265 (2007) hep-th/0611086.

[27] M. B. Green, J. G. Russo and P. Vanhove, "Ultraviolet properties of maximal supergravity," hep-th/0611273.

[28] G. Passarino and M. J. G. Veltman, "One Loop Corrections For E+ E- Annihilation Into Mu+ Mu- In The Weinberg Model," Nucl. Phys. B 160, 151 (1979).

[29] Z. Bern, N. E. J. Bjerrum-Bohr and D. C. Dunbar, "Inherited twistor-space structure of gravity loop amplitudes," JHEP 0505, 056 (2005) hep-th/0501137.

[30] N. E. J. Bjerrum-Bohr, D. C. Dunbar, H. Ita, W. B. Perkins and K. Risager, "The no-triangle hypothesis for N = 8 supergravity," JHEP 0612, 072 (2006) hep-th/0610043.

[31] E. Witten, "Perturbative gauge theory as a string theory in twistor space," Phys. Rev. D 66, 010001 (2002) hep-th/0312171.

[32] V. P. Nair, "A note on MHV amplitudes for gravitons," Phys. Rev. D 71, 121701 (2005) hep-th/0501143.

[33] M. Abou-Zeid, C. M. Hull and L. J. Mason, "Einstein supergravity and new twistor string theories," hep-th/0606272.

[34] N. E. J. Bjerrum-Bohr, D. C. Dunbar, H. Ita, W. B. Perkins and K. Risager, "MHV-vertices for gravity amplitudes," JHEP 0601, 009 (2006) hep-th/0509016.

[35] K. Risager, "A direct proof of the CSW rules," JHEP 0512, 003 (2005) hep-th/0508206.

[36] M. Bianchi, H. Elvang and D. Z. Freedman, JHEP 0809 (2008) 063 [arXiv:0805.0757 [hep-th]].

[37] Z. Bern, L. J. Dixon and D. A. Kosower, "On-shell recurrence relations for one-loop QCD amplitudes," Phys. Rev. D 71, 105013 (2005) hep-th/0501240.

[38] F. A. Berends, R. Kleiss, P. De Causmaecker, R. Gastmans and T. T. Wu, "Single Bremsstrahlung Processes In Gauge Theories," Phys. Lett. B 103, 124 (1981).

[39] P. De Causmaecker, R. Gastmans, W. Troost and T. T. Wu, "Multiple Bremsstrahlung In Gauge Theories At High-Energies. 1. General Formalism For Quantum Electrodynamics," Nucl. Phys. B 206, 53 (1982).

[40] R. Kleiss and W. J. Stirling, "Spinor Techniques For Calculating P Anti-P $\rightarrow$ W+- / Z0 + Jets," Nucl. Phys. B 262, 235 (1985).

[41] Z. Bern, "Perturbative quantum gravity and its relation to gauge theory," Living Rev. Rel. 5, 5 (2002) gr-qc/0206071. 
[42] N. Arkani-Hamed and J. Kaplan, "On Tree Amplitudes in Gauge Theory and Gravity," JHEP 0804, 076 (2008) [arXiv:0801.2385 [hep-th]]. 